\title{
A THREE-LEVEL BDDC ALGORITHM FOR MORTAR DISCRETIZATIONS*
}

\author{
HYEA HYUN $\mathrm{KIM}^{\dagger}$ AND XUEMIN TU
}

\begin{abstract}
In this paper, a three-level balancing domain decomposition by constraints (BDDC) algorithm is developed for the solutions of large sparse algebraic linear systems arising from the mortar discretization of elliptic boundary value problems. The mortar discretization is considered on geometrically nonconforming subdomain partitions. In two-level BDDC algorithms, the coarse problem needs to be solved exactly. However, its size will increase with the increase of the number of the subdomains. To overcome this limitation, the three-level algorithm solves the coarse problem inexactly while a good rate of convergence is maintained. This is an extension of previous work: the three-level BDDC algorithms for standard finite element discretization. Estimates of the condition numbers are provided for the three-level BDDC method, and numerical experiments are also discussed.
\end{abstract}

Key words. mortar discretization, balancing domain decomposition by constraints, three-level, domain decomposition, coarse problem, condition number

AMS subject classifications. $65 \mathrm{~N} 30,65 \mathrm{~N} 55$

DOI. $10.1137 / 07069081 \mathrm{X}$

1. Introduction. Mortar methods were introduced by Bernardi, Maday, and Patera [3] to couple different approximations in different subdomains so as to obtain a good global approximate solution. They are useful for modeling multiphysics, adaptivity, problems with joints, and mesh generation for three-dimensional complex structures. The coupling between different subdomains in mortar methods is done by enforcing certain constraints on solutions across the subdomain interface using Lagrange multipliers. We call these constraints the mortar matching conditions.

Balancing domain decomposition by constraints (BDDC) methods were introduced and analyzed in $[9,23,22]$ for elliptic problems with standard finite element discretizations. These iterative methods are new versions of the balancing NeumannNeumann algorithms with a coarse problem given in terms of a set of primal constraints. Two-level BDDC methods have been extended to saddle point problems in $[19,10,28,30]$, indefinite problems in [18], nonsymmetric problems in [27], and the problems with mortar finite element discretization in $[13,14]$. The complicated geometrically nonconforming subdomain partition leads to a much larger coarse problem than that of the standard discretization. In the two-level BDDC algorithms, the coarse problems are generated and factored by direct solvers at the beginning of the computation. The coarse components can be a bottleneck of the algorithms if the number of the subdomains is large.

${ }^{*}$ Received by the editors May 8, 2007; accepted for publication (in revised form) November 30, 2008; published electronically April 16, 2009. The work of the authors was supported in part by the U.S. Department of Energy under contract DE-FC02-01ER25482.

http://www.siam.org/journals/sinum/47-2/69081.html

$\dagger$ Department of Mathematics, Chonnam National University, Youngbong-dong, Buk-gu, Gwangju 500-757, Korea (hyeahyun@gmail.com, hkim@jnu.ac.kr). This author's work was also supported in part by Chonnam National University, 2008.

${ }^{\ddagger}$ Department of Mathematics, University of California at Berkeley and Lawrence Berkeley National Laboratory, Berkeley, CA 94720-3840 (xuemin@math.berkeley.edu). This author's research was also supported in part by the Director, Office of Science, Advanced Scientific Computing Research, U.S. Department of Energy under contract DE-AC02-05CH11231.

1576 
Recently, there have been several papers about inexact solvers for BDDC algorithms with standard finite element discretization. In [32, 31], two three-level BDDC algorithms are introduced which solve the coarse problems inexactly by introducing an additional level. Inexact local solvers based on multigrid methods were introduced in [21]. In [11], several inexact solvers for both the coarse and local components are considered. An inexact dual-primal finite element tearing and interconnecting (FETI-DP) algorithm is also introduced in [16]. The connection between FETI-DP and BDDC algorithms has been discussed in $[22,20,4,6]$.

In this paper, we extend the algorithms in [32] to mortar finite element discretization with quite general subdomain partitions. We solve the coarse problem approximately by introducing an additional level and using the BDDC algorithm recursively. We decompose the whole domain into subdomains and then group several subdomains to subregions to obtain a subregion partition. The subdomain partition can be geometrically nonconforming (it does not need to form a triangulation of the original domain), and the subregions usually will be irregular (they may not have uniformly Lipschitz continuous boundaries). We assume that our subregions are uniform domains and apply the results developed for such irregular domains in [15] to our analysis. See [15] and the references therein for the definition of uniform domains.

We provide estimates of the condition number bounds of the system with the new preconditioners and show that a good rate of convergence can still be maintained. We note that we have to choose the edge average primal constraints in the mortar discretization due to the mortar matching conditions. The resulting coarse problems are different from the ones in [32], where the vertex primal constraints are used. This difference and the geometrically nonconforming subdomain partition need a more complicated analysis for the condition number bound. We also note that this analysis can be used for the three-level BDDC algorithms for standard finite element discretization with edge primal constraints chosen for two dimensions.

The rest of the paper is organized as follows. We first review a two-level BDDC method for mortar discretization briefly in section 2. A three-level BDDC method and the corresponding preconditioner $\widetilde{M}^{-1}$ are introduced in section 3 . We give some auxiliary results in section 4 . In section 5 , we provide an estimate of the condition number bound for the system with the preconditioner $\widetilde{M}^{-1}$ which is of the form $C(1+\log (\widehat{H} / H))^{2}(1+\log (H / h))^{2}$, where $\widehat{H}, H$, and $h$ are typical diameters of the subregions, subdomains, and elements, respectively; see section 3 for the definitions of subregions and subdomains. Finally, some numerical experiments are discussed in section 6 .

Throughout the paper, $C$ denotes a generic positive constant that does not depend on any mesh parameters and the problem coefficients.

\section{A two-level BDDC algorithm for mortar discretizations.}

2.1. A model problem and the mortar discretizations. We will consider a second order scalar elliptic problem in a two-dimensional region $\Omega$ : find $u \in H_{0}^{1}(\Omega)$ such that

$$
\int_{\Omega} \rho \nabla u \cdot \nabla v d x=\int_{\Omega} f v d x \quad \forall v \in H_{0}^{1}(\Omega)
$$

where $\rho(x)>0$ for all $x \in \Omega$ and $f \in L^{2}(\Omega)$.

We decompose $\Omega$ into $N$ nonoverlapping subdomains $\Omega_{i}$ with diameters $H_{i}$ and set $H=\max _{i} H_{i}$. We make the following assumption for our subdomain partition. 
Assumption 2.1. Subdomains are polygons, and each subdomain has comparable diameter to its neighbors.

The partition can be geometrically nonconforming, where a pair of subdomains can intersect only a part of a subdomain edge. In other words, the partition does not need to form a triangulation of $\Omega$. In the following, we will regard the edges as the interface between subdomains. We then define the interface of the subdomain partition by

$$
\Gamma=\bigcup_{i j} \bar{F}_{i j} \backslash \partial \Omega
$$

where

$$
F_{i j}=\partial \Omega_{i} \bigcap \partial \Omega_{j}
$$

A quasi-uniform triangulation is given for each subdomain. We introduce $\mathbf{W}^{(i)}$, the standard finite element space of continuous, piecewise linear functions associated with the given triangulation in $\Omega_{i}$. In addition, the functions in $\mathbf{W}^{(i)}$ vanish on $\partial \Omega$. We define the product space of subdomain finite element spaces by

$$
\mathbf{W}=\prod_{i} \mathbf{W}^{(i)} .
$$

Functions in $\mathbf{W}$ can be discontinuous across the subdomain interface $\Gamma$.

The mortar methods are nonconforming finite element methods. To find a good approximate solution, the mortar matching condition is enforced on functions in the space $\mathbf{W}$ across the subdomain interface by using suitable Lagrange multipliers. Optimal order of approximation has been proved for the elliptic problems in both two and three dimensions; see $[3,1,2]$. In [3], the error estimate for the mortar approximation was first proved for both geometrically conforming and nonconforming partitions.

To introduce Lagrange multiplier spaces, we first select nonmortar and mortar parts of the interface. Among the subdomain edges, we can select edges $F_{l}$ that provide a disjoint covering of the interface $\Gamma$ (see [25, section 4.1])

$$
\bigcup_{l} \bar{F}_{l}=\bar{\Gamma}, \quad F_{l} \cap F_{k}=\emptyset, l \neq k .
$$

Each $F_{l}$ is a full edge of a subdomain. We call these edges the nonmortar edges. Since the subdomain partition can be geometrically nonconforming, a single nonmortar edge $F_{l} \subset \partial \Omega_{i}$ may intersect several subdomain boundaries. This provides $F_{l}$ with a partition

$$
\bar{F}_{l}=\bigcup_{j} \bar{F}_{i j}, \quad F_{i j}=\partial \Omega_{i} \cap \partial \Omega_{j}
$$

We call these $F_{i j}$, the mortar edges, which are opposite to $F_{l}$ and can be only a part of a subdomain edge.

A dual or a standard Lagrange multiplier space $\mathbf{M}\left(F_{l}\right)$ is given for each nonmortar edge $F_{l} \subset \partial \Omega_{i}$. We define a space

$$
\stackrel{\circ}{\mathbf{W}}\left(F_{l}\right):=\left.\mathbf{W}^{(i)}\right|_{F_{l}} \cap H_{0}^{1}\left(F_{l}\right)
$$

that is the restriction of the finite element functions to the nonmortar edges and vanishes on the boundary of these edges. We require that the space $\mathbf{M}\left(F_{l}\right)$ has the same dimension as the space $\mathbf{W}_{\mathbf{W}}\left(F_{l}\right)$ and that it contains the constant functions. Constructions of such Lagrange multiplier spaces were first given in $[1,3]$ for standard 
Lagrange multiplier spaces and in $[33,34]$ for dual Lagrange multiplier spaces; see also [12]. We note that the basis functions $\left\{\psi_{k}\right\}_{k}$ of the Lagrange multiplier space $M\left(F_{l}\right)$ satisfy

$$
\sum_{k} \psi_{k}=1
$$

For $\left(w_{1}, \ldots, w_{N}\right) \in \mathbf{W}$, we define $\phi \in L^{2}\left(F_{l}\right)$ by $\phi=w_{j}$ on $F_{i j} \subset F_{l}$. The mortar matching condition in the geometrically nonconforming partition is then given by

$$
\int_{F_{l}}\left(w_{i}-\phi\right) \lambda d s=0 \quad \forall \lambda \in \mathbf{M}\left(F_{l}\right) \forall F_{l} .
$$

We further define the following two product spaces of the $\mathbf{M}\left(F_{l}\right)$ and $\stackrel{\circ}{\mathbf{W}}\left(F_{l}\right)$, respectively:

$$
\mathbf{M}=\prod_{l} \mathbf{M}\left(F_{l}\right) \text { and } \mathbf{W}_{n}=\prod_{l} \stackrel{\circ}{\mathbf{W}}\left(F_{l}\right) .
$$

The mortar discretization for problem (2.1) is to approximate the solution by Galerkin's method in the mortar finite element space

$$
\widehat{\mathbf{W}}:=\{w \in \mathbf{W}: w \text { satisfies the mortar matching condition }(2.3)\} .
$$

2.2. A two-level BDDC algorithm. In this subsection, we construct a twolevel BDDC algorithm for the mortar discretization as in [13]. We first derive the primal form of the mortar discretization and then introduce a BDDC preconditioner for the primal form.

We divide unknowns in the subdomain finite element space $\mathbf{W}^{(i)}$ into subdomain interior and interface parts. We then select primal unknowns among the interface unknowns and further decompose the interface unknowns into the primal and the rest, called dual unknowns:

$$
\mathbf{W}^{(i)}=\mathbf{W}_{I}^{(i)} \times \mathbf{W}_{\Gamma}^{(i)} \quad \text { and } \quad \mathbf{W}_{\Gamma}^{(i)}=\mathbf{W}_{\Pi}^{(i)} \times \mathbf{W}_{\Delta}^{(i)},
$$

where $I, \Gamma, \Pi$, and $\Delta$ denote the interior, interface, primal, and dual unknowns, respectively.

The primal unknowns are related to certain primal constraints selected from the mortar matching condition (2.3), and they result in a coarse component of the BDDC preconditioner. A proper selection of such constraints is important to obtain a scalable BDDC algorithm. We consider $\left\{\psi_{i j, k}\right\}_{k}$, the basis functions in $\mathbf{M}\left(F_{l}\right)$ that are supported in $\bar{F}_{i j}$, and introduce

$$
\psi_{i j}=\sum_{k} \psi_{i j, k}
$$

Assumption 2.2. There is at least one basis function $\psi_{i j, k}$ whose support belongs to $\bar{F}_{i j}$.

We introduce the trace space of $\mathbf{W}$ on the subdomain boundaries

$$
\mathbf{W}_{\Gamma}=\prod_{i=1}^{N} \mathbf{W}_{\Gamma}^{(i)} .
$$

Copyright $@$ by SIAM. Unauthorized reproduction of this article is prohibited. 
We select the primal constraints for $\left(w_{1}, \ldots, w_{N}\right) \in \mathbf{W}_{\Gamma}$ over each interface $F_{i j}$ to satisfy

$$
\int_{F_{i j}}\left(w_{i}-w_{j}\right) \psi_{i j} d s=0
$$

In more detail, the primal unknowns associated to these constraints will be defined by

$$
u_{\pi}=\frac{\int_{F_{i j}} w_{i} \psi_{i j} d s}{\int_{F_{i j}} \psi_{i j} d s}=\frac{\int_{F_{i j}} w_{j} \psi_{i j} d s}{\int_{F_{i j}} \psi_{i j} d s} .
$$

In the case of a geometrically conforming partition, i.e., when $F_{i j}$ is a full edge of two subdomains, the above constraints are the regular edge average matching condition because $\psi_{i j}=1$, the sum of all Lagrange multiplier basis functions $\left\{\psi_{i j, k}\right\}_{k}$ provided for $F_{i j}$; see (2.6) and (2.2).

We make the primal constraints explicit by a change of variables; see $[17$, section $6.2]$, [20, section 2.3], and [13, section 2.2]. We then separate the unknowns in the space $\mathbf{W}^{(i)}$ as described in (2.5). We will also assume that all of the matrices and vectors are written in terms of the new unknowns.

Throughout this paper, we use the notation $\mathbf{V}$ for the product space of local finite element spaces $\mathbf{V}^{(i)}$. In addition, we use the notation $\widehat{\mathbf{V}}$ for a subspace of $\mathbf{V}$ satisfying the mortar matching condition (or pointwise continuity condition) across the subdomain interface and the notation $\widetilde{\mathbf{V}}$ for a subspace of $\mathbf{V}$ satisfying only the primal constraints. For example, we can represent the space

$$
\widetilde{\mathbf{W}}_{\Gamma}=\left\{w \in \mathbf{W}_{\Gamma}: w \text { satisfies the primal constraints }(2.7)\right\}
$$

in the following way:

$$
\widetilde{\mathbf{W}}_{\Gamma}=\mathbf{W}_{\Delta} \times \widehat{\mathbf{W}}_{\Pi} .
$$

We further decompose the dual unknowns into the unknowns in the nonmortar part and the rest:

$$
\mathbf{W}_{\Delta}=\mathbf{W}_{\Delta, n} \times \mathbf{W}_{\Delta, m},
$$

where $n$ and $m$ denote unknowns in each part, respectively.

The matrix representation of the mortar matching condition (2.3) on functions in the space $\widetilde{\mathbf{W}}_{\Gamma}$ can be written as

$$
B_{n} w_{n}+B_{m} w_{m}+B_{\Pi} w_{\Pi}=0 .
$$

Here we enforced the mortar matching condition using a reduced Lagrange multiplier space, since the functions in the space $\widetilde{\mathbf{W}}_{\Gamma}$ satisfy the primal constraints selected from the mortar matching condition (2.3). The reduced Lagrange multiplier space is obtained after eliminating one basis function among $\left\{\psi_{i j, k}\right\}_{k}$ for each $F_{i j} \subset F_{l}$ so that the matrix $B_{n}$ in (2.9) is invertible. The unknowns $w_{n}$ are then determined by the other unknowns $\left(w_{m}, w_{\Pi}\right)$, which are called the genuine unknowns. We define the space of genuine unknowns by

$$
\mathbf{W}_{G}=\mathbf{W}_{\Delta, m} \times \widehat{\mathbf{W}}_{\Pi}
$$


and define the mortar map by

$$
\widetilde{R}_{\Gamma}=\left(\begin{array}{cc}
-B_{n}^{-1} B_{m} & -B_{n}^{-1} B_{\Pi} \\
I & 0 \\
0 & I
\end{array}\right)
$$

that maps the genuine unknowns in $\mathbf{W}_{G}$ into the unknowns in $\widetilde{\mathbf{W}}_{\Gamma}$ which satisfy the mortar matching condition (2.9). In the following, we will regard $\mathbf{W}_{G}$ as the space $\widehat{\mathbf{W}}_{\Gamma}$ and regard $\widetilde{R}_{\Gamma}$ as an extension from $\widehat{\mathbf{W}}_{\Gamma}$ to the space $\widetilde{\mathbf{W}}_{\Gamma}$ to be consistent with notations of the three-level algorithm.

To derive the linear system of the mortar discretization, we introduce several matrices. The matrix $S_{\Gamma}^{(i)}$ is the local Schur complement matrix obtained by eliminating the subdomain interior unknowns

$$
S_{\Gamma}^{(i)}=K_{\Gamma \Gamma}^{(i)}-K_{\Gamma I}^{(i)}\left(K_{I I}^{(i)}\right)^{-1}\left(K_{\Gamma I}^{(i)}\right)^{T}
$$

where $K^{(i)}$ is the local stiffness matrix ordered as follows:

$$
K^{(i)}=\left(\begin{array}{cc}
K_{I I}^{(i)} & K_{I \Gamma}^{(i)} \\
K_{\Gamma I}^{(i)} & K_{\Gamma \Gamma}^{(i)}
\end{array}\right)=\left(\begin{array}{ccc}
K_{I I}^{(i)} & K_{I \Delta}^{(i)} & K_{I \Pi}^{(i)} \\
K_{\Delta I}^{(i)} & K_{\Delta \Delta}^{(i)} & K_{\Delta \Pi}^{(i)} \\
K_{\Pi I}^{(i)} & K_{\Pi \Delta}^{(i)} & K_{\Pi \Pi}^{(i)}
\end{array}\right) .
$$

We define extensions $\widetilde{R}_{\Gamma}$ and $R_{\Gamma}$ by

$$
\widehat{\mathbf{W}}_{\Gamma} \stackrel{\widetilde{R}_{\Gamma}}{\longrightarrow} \widetilde{\mathbf{W}}_{\Gamma} \stackrel{R_{\Gamma}}{\longrightarrow} \mathbf{W}_{\Gamma}
$$

where $\widetilde{R}_{\Gamma}$ is the mortar map in (2.10) and $R_{\Gamma}$ is the product of restriction maps

$$
R_{\Gamma}^{(i)}: \widetilde{\mathbf{W}}_{\Gamma} \rightarrow \mathbf{W}_{\Gamma}^{(i)}
$$

We next introduce the matrices $S_{\Gamma}$ and $\widetilde{S}_{\Gamma}$, the block diagonal matrix and the partially assembled matrix at the primal unknowns, respectively, as

$$
S_{\Gamma}=\operatorname{diag}_{i}\left(S_{\Gamma}^{(i)}\right) \text { and } \widetilde{S}_{\Gamma}=R_{\Gamma}^{T} S_{\Gamma} R_{\Gamma} .
$$

The linear system of the mortar discretization is then written as follows: find $\mathbf{u}_{G} \in \widehat{\mathbf{W}}_{\Gamma}$ such that

$$
\widetilde{R}_{\Gamma}^{T} \widetilde{S}_{\Gamma} \widetilde{R}_{\Gamma} \mathbf{u}_{G}=\widetilde{R}_{\Gamma}^{T} \mathbf{g}_{G}
$$

where $\mathbf{g}_{G} \in \widehat{\mathbf{W}}_{\Gamma}$ is the part of genuine unknowns of $\mathbf{g}_{\Gamma} \in \mathbf{W}_{\Gamma}$ and $\mathbf{g}_{\Gamma}$ is given by

$$
\left.\mathbf{g}_{\Gamma}\right|_{\partial \Omega_{i}}=\mathbf{g}_{\Gamma}^{(i)}=\mathbf{f}_{\Gamma}^{(i)}-K_{\Gamma I}^{(i)}\left(K_{I I}^{(i)}\right)^{-1} \mathbf{f}_{I}^{(i)},
$$

where $\mathbf{f}^{(i)}=\left(\begin{array}{l}\mathbf{f}_{\mathbf{f}_{\Gamma}^{(i)}}^{(i)} \\ \mathbf{f}_{\Gamma}^{(i)}\end{array}\right)$, the local load vector.

In the two-level BDDC algorithm in [13], we solve (2.11) using a preconditioner $M^{-1}$ of the form

$$
M^{-1}=\widetilde{R}_{D, \Gamma}^{T} \widetilde{S}_{\Gamma}^{-1} \widetilde{R}_{D, \Gamma},
$$

Copyright $@$ by SIAM. Unauthorized reproduction of this article is prohibited. 
where the weighted extension operator $\widetilde{R}_{D, \Gamma}$ is given by

$$
\widetilde{R}_{D, \Gamma}=D \widetilde{R}_{\Gamma}=\left(\begin{array}{ccc}
\mathbf{D}_{n} & \mathbf{0} & \mathbf{0} \\
\mathbf{0} & \mathbf{D}_{m} & \mathbf{0} \\
\mathbf{0} & \mathbf{0} & \mathbf{D}_{\Pi}
\end{array}\right) \widetilde{R}_{\Gamma}, \quad \mathbf{D}_{n}=\mathbf{0}, \mathbf{D}_{m}=\mathbf{I}, \mathbf{D}_{\Pi}=\mathbf{I} .
$$

We call $M^{-1}$ the Neumann-Dirichlet preconditioner. The weight factor $D$ is determined to be zero at the nonmortar interfaces and to be one otherwise. This type of weight was shown to be the most efficient for the elliptic problems with jump coefficients $\rho_{i}$ when the part with smaller $\rho_{i}$ is selected to be the nonmortar part; see [7].

Assumption 2.3. We select the nonmortar and mortar parts of the interface $F_{i j}\left(=\partial \Omega_{i} \bigcap \partial \Omega_{j}\right)$ to satisfy

$$
\rho_{i} \leq \rho_{j},
$$

where $\Omega_{i}$ is the nonmortar part and $\Omega_{j}$ is the mortar part.

Using a block Cholesky factorization, we obtain

$$
\widetilde{S}_{\Gamma}^{-1}=R_{\Gamma \Delta}^{T}\left(\sum_{i=1}^{N}\left(\mathbf{0} R_{\Delta}^{(i)^{T}}\right)\left(\begin{array}{cc}
K_{I I}^{(i)} & K_{I \Delta}^{(i)} \\
K_{\Delta I}^{(i)} & K_{\Delta \Delta}^{(i)}
\end{array}\right)^{-1}\left(\begin{array}{c}
\mathbf{0} \\
R_{\Delta}^{(i)}
\end{array}\right)\right) R_{\Gamma \Delta}+\Phi S_{\Pi}^{-1} \Phi^{T}
$$

where the restrictions $R_{\Gamma \Delta}$ and $R_{\Delta}^{(i)}$ are defined by

$$
R_{\Gamma \Delta}: \widetilde{\mathbf{W}}_{\Gamma} \rightarrow \mathbf{W}_{\Delta} \text { and } R_{\Delta}^{(i)}: \mathbf{W}_{\Delta} \rightarrow \mathbf{W}_{\Delta}^{(i)} .
$$

Here $\Phi$ is the matrix whose columns are the coarse basis functions with minimal energy

$$
\Phi=R_{\Gamma \Pi}^{T}-R_{\Gamma \Delta}^{T} \sum_{i=1}^{N}\left(\mathbf{0} R_{\Delta}^{(i)^{T}}\right)\left(\begin{array}{cc}
K_{I I}^{(i)} & K_{I \Delta}^{(i)} \\
K_{\Delta I}^{(i)} & K_{\Delta \Delta}^{(i)}
\end{array}\right)^{-1}\left(\begin{array}{c}
K_{\Pi I}^{(i)^{T}} \\
K_{\Pi \Delta}^{(i)^{T}}
\end{array}\right) R_{\Pi}^{(i)},
$$

where $R_{\Gamma \Pi}$ and $R_{\Pi}^{(i)}$ are the restrictions

$$
R_{\Gamma \Pi}: \widetilde{\mathbf{W}}_{\Gamma} \rightarrow \widehat{\mathbf{W}}_{\Pi} \text { and } R_{\Pi}^{(i)}: \widehat{\mathbf{W}}_{\Pi} \rightarrow \mathbf{W}_{\Pi}^{(i)} .
$$

The coarse level problem matrix $S_{\Pi}$ is determined by

$$
S_{\Pi}=\sum_{i=1}^{N} R_{\Pi}^{(i)^{T}}\left\{K_{\Pi \Pi}^{(i)}-\left(\begin{array}{ll}
K_{\Pi I}^{(i)} & K_{\Pi \Delta}^{(i)}
\end{array}\right)\left(\begin{array}{cc}
K_{I I}^{(i)} & K_{I \Delta}^{(i)} \\
K_{\Delta I}^{(i)} & K_{\Delta \Delta}^{(i)}
\end{array}\right)^{-1}\left(\begin{array}{c}
K_{\Pi I}^{(i)^{T}} \\
K_{\Pi \Delta}^{(i)^{T}}
\end{array}\right)\right\} R_{\Pi}^{(i)},
$$

which is obtained by assembling subdomain matrices; for additional details, see $[9$, 20, 23]. Therefore, the preconditioner $M^{-1}$ contains local components and a coarse component that involve solving the Neumann problems in each subdomain and solving the coarse problem with the matrix $S_{\Pi}$, respectively.

From [13, Theorem 4.7], we know that for any $\mathbf{u}_{\Gamma} \in \widehat{\mathbf{W}}_{\Gamma}$,

$$
\mathbf{u}_{\Gamma}^{T} M \mathbf{u}_{\Gamma} \leq \mathbf{u}_{\Gamma}^{T} \widetilde{R}_{\Gamma}^{T} \widetilde{S}_{\Gamma} \widetilde{R}_{\Gamma} \mathbf{u}_{\Gamma} \leq C(1+\log (H / h))^{2} \mathbf{u}_{\Gamma}^{T} M \mathbf{u}_{\Gamma} .
$$



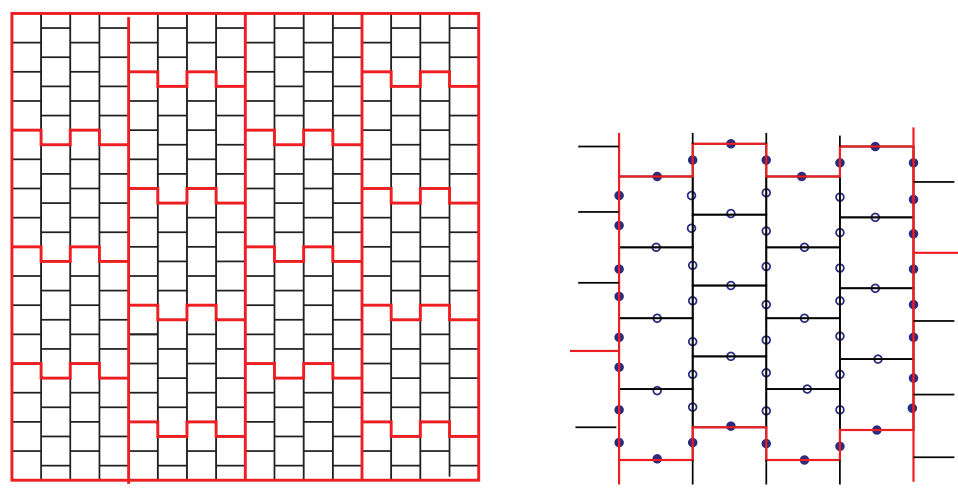

FIG. 1. A subregion partition (left) and unknowns at a subregion (right) when $\widehat{H} / H=4$; the small rectangles are subdomains, the white nodes designate primal unknowns at the interior of the subregion, and the black nodes designate primal unknowns on the subregion boundary.

3. A three-level BDDC method. In the three-level algorithms, as in [32, 31], we will not factor the coarse problem matrix $S_{\Pi}$ defined in $(2.15)$ by a direct solver. Instead, we will introduce another level and solve the coarse problem approximately on this level by using ideas similar to those for the two-level preconditioners.

Let subregion $\Omega^{j}$ be a union of $N_{j}$ subdomains $\Omega_{i}^{j}$ with diameters $H_{i}^{(j)}$, and then we obtain a subregion partition $\left\{\Omega^{j}\right\}_{j=1}^{N_{c}}$. We make the following assumption on our subregions; see [15] and the references therein for the definition of uniform domains.

Assumption 3.1. The subregions are uniform domains.

We denote by $\widehat{H}^{(j)}$ the diameter of the subregion $\Omega^{j}$. Let $\widehat{H}=\max _{j} \widehat{H}^{(j)}$ and $H=\max _{i, j} H_{i}^{(j)}$. Then $N$, the total number of subdomains, can be written as $N=N_{1}+\cdots+N_{N_{c}}$. An example of a subregion partition that is obtained from a geometrically nonconforming subdomain partition is shown in Figure 1. In the following, we will use a superscript for the subregion index and a subscript for the subdomain index, for example, $\Omega^{j}$ and $\Omega_{i}$ for subregions and subdomains, respectively. For subdomains in the subregion $\Omega^{j}$, we use the notation $\Omega_{i}^{j}$.

In the subregion partition, we define edges as the intersection of two subregions and vertices as the intersection of more than two subregions, similar to [26, Definition 4.1]. In addition, the finite element spaces for the subregions are given by the primal unknowns of the two-level algorithm so that the subregion partition is equipped with a conforming finite element space for which the unknowns match across the subregion interface. On this new level, the mortar discretization is no longer relevant. We can then develop the theory and algorithm for the subregion partition as in the standard BDDC algorithm for conforming finite element discretizations. However, we need to construct appropriate finite element spaces for the subregions equipped with the primal unknowns to provide the condition number bound.

We obtain the subregion matrix $S_{\Pi}^{(j)}$ by assembling the coarse problem matrices of the subdomains $\Omega_{i}^{j} \subset \Omega^{j}$ :

$$
S_{\Pi}^{(j)}=\sum_{i=1}^{N_{j}} R_{\Pi}^{(i)^{T}}\left\{K_{\Pi \Pi}^{(i)}-\left(\begin{array}{ll}
K_{\Pi I}^{(i)} & K_{\Pi \Delta}^{(i)}
\end{array}\right)\left(\begin{array}{cc}
K_{I I}^{(i)} & K_{I \Delta}^{(i)} \\
K_{\Delta I}^{(i)} & K_{\Delta \Delta}^{(i)}
\end{array}\right)^{-1}\left(\begin{array}{c}
K_{\Pi I}^{(i)^{T}} \\
K_{\Pi \Delta}^{(i)^{T}}
\end{array}\right)\right\} R_{\Pi}^{(i)},
$$

where $R_{\Pi}^{(i)}:\left.\widehat{W}_{\Pi}\right|_{\Omega^{j}} \rightarrow \widehat{W}_{\Pi}^{(i)}$ is the restriction of primal unknowns in the subregion 
$\Omega^{j}$ to the subdomain $\Omega_{i}^{j}$. We note that the global coarse problem matrix $S_{\Pi}$ can be assembled from the $S_{\Pi}^{(j)}$ of each subregion.

We will build a BDDC preconditioner for the problem $S_{\Pi}$ following the same construction as in the two-level algorithm for standard conforming finite element discretizations. In the following, we introduce the same finite element spaces as in the previous section except that they are based on the subregion partition and the subregion unknowns. We will use the subscript $c$ to denote those unknowns, function spaces, and matrices related to the subregion level. For example, $\mathbf{W}_{c}^{(j)}$ denotes the discrete space for the subregion $\Omega^{j}$. It consists of the primal unknowns of the two-level algorithm contained in the subregion $\Omega^{j}$.

Let $\Gamma_{c}$ be the interface between the subregions and $\Gamma_{c} \subset \Gamma$. We then decompose the subregion unknowns into subregion interior and interface unknowns and further decompose the interface unknowns into primal and dual unknowns

$$
\mathbf{W}_{c}^{(j)}=\mathbf{W}_{I_{c}}^{(j)} \times \mathbf{W}_{\Gamma_{c}}^{(j)} \quad \text { and } \quad \mathbf{W}_{\Gamma_{c}}^{(j)}=\mathbf{W}_{\Pi_{c}}^{(j)} \times \mathbf{W}_{\Delta_{c}}^{(j)} .
$$

Here the average constraints on subregion edges have been selected as the primal constraints and we have changed the variables to make the primal constraints explicit. Similarly, we define the product space $\mathbf{W}_{\Gamma_{c}}$, its subspaces $\widehat{\mathbf{W}}_{\Gamma_{c}}$ and $\widetilde{\mathbf{W}}_{\Gamma_{c}}$, and the extensions

$$
\widehat{\mathbf{W}}_{\Gamma_{c}} \stackrel{\widetilde{R}_{\Gamma_{c}}}{\longrightarrow} \widetilde{\mathbf{W}}_{\Gamma_{c}} \stackrel{R_{\Gamma_{c}}}{\longrightarrow} \mathbf{W}_{\Gamma_{c}}
$$

We note that $\widehat{\mathbf{W}}_{\Gamma_{c}}$ is the space of vectors of unknowns that have the same values across the subregion interface and $\widetilde{\mathbf{W}}_{\Gamma_{c}}$ is the space of vectors of unknowns that have the same values at the subregional primal unknowns and can have different values at the other interface unknowns.

We define our three-level preconditioner $\widetilde{M}^{-1}$ by

$\widetilde{R}_{D, \Gamma}^{T}\left\{R_{\Gamma \Delta}^{T}\left(\sum_{i=1}^{N}\left(\mathbf{0} R_{\Delta}^{(i)^{T}}\right)\left(\begin{array}{cc}K_{I I}^{(i)} & K_{I \Delta}^{(i)} \\ K_{\Delta I}^{(i)} & K_{\Delta \Delta}^{(i)}\end{array}\right)^{-1}\left(\begin{array}{c}\mathbf{0} \\ R_{\Delta}^{(i)}\end{array}\right)\right) R_{\Gamma \Delta}+\Phi M_{\Pi}^{-1} \Phi^{T}\right\} \widetilde{R}_{D, \Gamma}$,

where $M_{\Pi}^{-1}$ is an approximation of $S_{\Pi}^{-1}$; see (2.14). In other words, for a given $\mathbf{\Psi} \in \widehat{\mathbf{W}}_{c}$, we compute $\mathbf{z}=M_{\Pi}^{-1} \boldsymbol{\Psi}$ instead of $\mathbf{y}=S_{\Pi}^{-1} \mathbf{\Psi}$.

We now introduce the approximation $M_{\Pi}^{-1}$ in detail. We first order the unknowns $\mathbf{y} \in \widehat{\mathbf{W}}_{c}$ into subregion interior and interface unknowns

$$
\mathbf{y}=\left(\mathbf{y}_{I_{c}}^{(1)}, \ldots, \mathbf{y}_{I_{c}}^{\left(N_{c}\right)}, \mathbf{y}_{\Gamma_{c}}\right)^{T} .
$$

We then write the problem $S_{\Pi} \mathbf{y}=\mathbf{\Psi}$ as

$$
\left(\begin{array}{cccc}
S_{\Pi_{I_{c} I_{c}}}^{(1)} & \mathbf{0} & \mathbf{0} & S_{\Pi_{\Gamma_{c} I_{c}}}^{(1)^{T}} R_{\Gamma_{c}}^{(1)} \\
\mathbf{0} & \ddots & \mathbf{0} & \vdots \\
\mathbf{0} & \mathbf{0} & S_{\Pi_{I_{c} I_{c}}}^{\left(N_{c}\right)} & S_{\Pi_{\Gamma_{c} I_{c}}}^{\left(N_{c}\right)^{T}} R_{\Gamma_{c}}^{\left(N_{c}\right)} \\
R_{\Gamma_{c}}^{(1)^{T}} S_{\Pi_{\Gamma_{c} I_{c}}}^{(1)} & \cdots & R_{\Gamma_{c}}^{\left(N_{c}\right)^{T}} S_{\Pi_{\Gamma_{c} I_{c}}}^{\left(N_{c}\right)} & S_{\Pi_{\Gamma_{c} \Gamma_{c}}}
\end{array}\right)\left(\begin{array}{c}
\mathbf{y}_{I_{c}}^{(1)} \\
\vdots \\
\mathbf{y}_{I_{c}}^{\left(N_{c}\right)} \\
\mathbf{y}_{\Gamma_{c}}
\end{array}\right)=\left(\begin{array}{c}
\boldsymbol{\Psi}_{I_{c}}^{(1)} \\
\vdots \\
\mathbf{\Psi}_{I_{c}}^{\left(N_{c}\right)} \\
\boldsymbol{\Psi}_{\Gamma_{c}}
\end{array}\right)
$$


where $R_{\Gamma_{c}}^{(j)}$ is the restriction and $S_{\Pi_{\Gamma_{c} \Gamma_{c}}}$ is the fully assembled matrix at the subregion interface

$$
R_{\Gamma_{c}}^{(j)}: \widehat{\mathbf{W}}_{\Gamma_{c}} \rightarrow \mathbf{W}_{\Gamma_{c}}^{(j)} \quad \text { and } \quad S_{\Pi_{\Gamma_{c} \Gamma_{c}}}=\sum_{j=1}^{N_{c}} R_{\Gamma_{c}}^{(j)^{T}} S_{\Pi_{\Gamma_{c} \Gamma_{c}}}^{(j)} R_{\Gamma_{c}}^{(j)}
$$

Here we solve for $\mathbf{y}_{I_{c}}^{(j)}$

$$
\mathbf{y}_{I_{c}}^{(j)}=S_{\Pi_{I_{c} I_{c}}}^{(j)^{-1}}\left(\mathbf{\Psi}_{I_{c}}^{(j)}-S_{\Pi_{\Gamma_{c} I_{c}}}^{(j)^{T}} R_{\Gamma_{c}}^{(j)} \mathbf{y}_{\Gamma_{c}}\right)
$$

and obtain the interface problem

$$
\left(\sum_{j=1}^{N_{c}} R_{\Gamma_{c}}^{(j)^{T}}\left(S_{\Pi_{\Gamma_{c} \Gamma_{c}}}^{(j)}-S_{\Pi_{\Gamma_{c} I_{c}}}^{(j)} S_{\Pi_{I_{c} I_{c}}}^{(j)^{-1}} S_{\Pi_{\Gamma_{c} I_{c}}}^{(j)^{T}}\right) R_{\Gamma_{c}}^{(j)}\right) \mathbf{y}_{\Gamma_{c}}=\mathbf{h}_{\Gamma_{c}},
$$

where

$$
\mathbf{h}_{\Gamma_{c}}=\boldsymbol{\Psi}_{\Gamma_{c}}-\sum_{j=1}^{N_{c}} R_{\Gamma_{c}}^{(j)^{T}} S_{\Pi_{\Gamma_{c} I_{c}}}^{(j)} S_{\Pi_{I_{c} I_{c}}}^{(j)^{-1}} \boldsymbol{\Psi}_{I_{c}}^{(j)} .
$$

We denote by $T^{(j)}$ the Schur complement of $S_{\Pi}^{(j)}$,

$$
T^{(j)}=S_{\Pi_{\Gamma_{c} \Gamma_{c}}}^{(j)}-S_{\Pi_{\Gamma_{c} I_{c}}}^{(j)}\left(S_{\Pi_{I_{c} I_{c}}}^{(j)}\right)^{-1}\left(S_{\Pi_{\Gamma_{c} I_{c}}}^{(j)}\right)^{T}
$$

and define the block diagonal matrix

$$
T=\operatorname{diag}_{j}\left(T^{(j)}\right) .
$$

We then introduce the partially assembled matrix and the fully assembled matrix

$$
\widetilde{T}=R_{\Gamma_{c}}^{T} T R_{\Gamma_{c}} \text { and } \widehat{T}=\widetilde{R}_{\Gamma_{c}}^{T} \widetilde{T} \widetilde{R}_{\Gamma_{c}},
$$

respectively, using the extensions $R_{\Gamma_{c}}$ and $\widetilde{R}_{\Gamma_{c}}$ defined in (3.1). The reduced subregional interface problem (3.5) is then written as follows: find $\mathbf{y}_{\Gamma_{c}} \in \widehat{\mathbf{W}}_{\Gamma_{c}}$ such that

$$
\widetilde{R}_{\Gamma_{c}}^{T} \widetilde{T}_{\Gamma_{c}} \mathbf{y}_{\Gamma_{c}}=\mathbf{h}_{\Gamma_{c}} .
$$

When using the three-level preconditioner $\widetilde{M}^{-1}$, we do not solve (3.8) exactly. Instead, we replace $\mathbf{y}_{\Gamma_{c}}$ by $\mathbf{z}_{\Gamma_{c}}$, where

$$
\mathbf{z}_{\Gamma_{c}}=\widetilde{R}_{D, \Gamma_{c}}^{T} \widetilde{T}^{-1} \widetilde{R}_{D, \Gamma_{c}} \mathbf{h}_{\Gamma_{c}} .
$$

Here $\widetilde{R}_{D, \Gamma_{c}}$ is the scaled extension such that $\widetilde{R}_{D, \Gamma_{c}}=D \widetilde{R}_{\Gamma_{c}}$. The three-level coarse problem appearing in the computation of $\widetilde{T}^{-1}$ is solved quite cheaply compared to that of the two-level algorithm, since its size is much smaller than that of the twolevel algorithm. The weight factor $D$ has the value 1 as its diagonal components corresponding to the global primal unknowns in $\widehat{\mathbf{W}}_{\Pi_{c}}$ and the following values for the other diagonal components:

$$
\delta_{c, j}^{\dagger}(x)=\frac{\rho_{j}^{\gamma}(x)}{\sum_{i \in \mathcal{N}_{x}} \rho_{i}^{\gamma}(x)}, \quad x \in n\left(\mathbf{W}_{\Delta_{c}}^{(j)}\right)
$$

Copyright $@$ by SIAM. Unauthorized reproduction of this article is prohibited. 
where $\gamma \in[1 / 2, \infty)$ and $n\left(\mathbf{W}_{\Delta_{c}}^{(j)}\right)$ denotes the set of nodes in the finite element space $\mathbf{W}_{\Delta_{c}}^{(j)}$. In addition, $\mathcal{N}_{x}$ is the set of the subregion indices $i$ such that $x \in n\left(\mathbf{W}_{\Delta_{c}}^{(i)}\right)$ and $\rho_{i}(x)$ is the coefficient of (2.1) at $x$ in the subregion $\Omega^{i}$. In our theory, $\rho_{i}(x)$ is a positive constant in the subregion $\Omega^{i}$.

Assumption 3.2. $\rho_{i}(x)$ is a positive constant in each subregion $\Omega^{i}$.

We then compute $\mathbf{z}_{I_{c}}^{(j)}$ from $\mathbf{z}_{\Gamma_{c}}$ as in (3.4):

$$
\mathbf{z}_{I_{c}}^{(j)}=S_{\Pi_{I_{c} I_{c}}}^{(j)^{-1}}\left(\Psi_{I_{c}}^{(j)}-S_{\Pi_{I_{c} \Gamma_{c}}}^{(j)} R_{\Gamma_{c}}^{(j)} \mathbf{z}_{\Gamma_{c}}\right) .
$$

As a result, we obtain $\mathbf{z}=M_{\Pi}^{-1} \boldsymbol{\Psi}$, the solution of the inexact coarse problem for a given $\boldsymbol{\Psi}$.

Let $\langle u, v\rangle$ denote the $l^{2}$-inner product for vectors $u$ and $v$. We summarize our three-level algorithm equipped with the preconditioner $\widetilde{M}^{-1}$ in (3.2) as follows:

Let $A=\widetilde{R}_{\Gamma}^{T} \widetilde{S}_{\Gamma} \widetilde{R}_{\Gamma}, b=\widetilde{R}_{\Gamma}^{T} \boldsymbol{g}_{G}$, and $T O L$ be given.

Step 1. Start with initial $x_{0}$, compute residual $r_{0}=b-A x_{0}$, and set $k=0$.

Step 2. while $\left(\left\|r_{k}\right\| /\left\|r_{0}\right\|>T O L\right)$

Step 2.1. $z_{k}=\widetilde{M}^{-1} r_{k}$

Step 2.2. $k=k+1$

Step 2.3. if $(k \geq 2)$

$$
\begin{aligned}
& \beta_{k}=\left\langle z_{k-1}, r_{k-1}\right\rangle /\left\langle z_{k-2}, r_{k-2}\right\rangle \\
& d_{k}=z_{k-1}+\beta_{k} d_{k-1} \\
& \text { else } \\
& \beta_{1}=0, d_{1}=z_{0}
\end{aligned}
$$

end if

Step 2.4. $\alpha_{k}=\left\langle z_{k-1}, r_{k-1}\right\rangle /\left\langle A d_{k}, d_{k}\right\rangle$

Step 2.5. Compute $x_{k}=x_{k-1}+\alpha_{k} d_{k}$

Step 2.6. Compute $r_{k}=b-A x_{k}$

end while

Step 3. $x=x_{k}$ is the required solution.

In the two-level algorithm, $\widetilde{M}^{-1}$ in Step 2.1 is replaced by the two-level preconditioner $M^{-1}$; see (2.12). From (2.12) and (2.14), we know that we need to solve subdomain local problems and one coarse problem exactly when we apply $M^{-1}$ to a vector in Step 2.1. When we use our three-level preconditioner $\widetilde{M}^{-1}$ in Step 2.1, we solve the subdomain local problems exactly as in the two-level algorithm; see (3.2). We do not solve the coarse problem exactly. Instead, we apply the standard two-level BDDC preconditioner to solve the coarse problem. In other words, we use (3.9) and (3.11), which will need to solve a subregion coarse problem and subregion local problems exactly. We note that the size of the subregion coarse problem is much smaller than that of the two-level coarse problem.

4. Some auxiliary results. In this section, we will collect a number of results which are needed in our theory.

In the following, the notation $f=O(g)$ means that there exist positive constants $c$ and $C$, independent of $H$ and $h$, such that

$$
c g \leq f \leq C g .
$$

Let $E$ be an edge of a subdomain $\Omega_{i}$. We introduce a Sobolev space $H_{00}^{1 / 2}(E)$ as

$$
H_{00}^{1 / 2}(E)=\left\{v \in L^{2}(E): \widetilde{v} \in H^{1 / 2}\left(\partial \Omega_{i}\right)\right\} .
$$


Here $\widetilde{v}$ is the zero extension of $v$ to the subdomain boundary. The norm is given by

$$
\|v\|_{H_{00}^{1 / 2}(E)}^{2}=|v|_{H^{1 / 2}(E)}^{2}+\int_{E} \frac{v(x)^{2}}{\operatorname{dist}(x, \partial E)} d s(x),
$$

where

$$
|v|_{H^{1 / 2}(E)}^{2}=\int_{E} \int_{E} \frac{|v(x)-v(y)|^{2}}{|x-y|^{2}} d s(x) d s(y) .
$$

Lemma 4.1. Given a function $g(x)=x(H-x)$ defined on $[0, H]$, we consider a nodal interpolant $g^{h}(x)=I^{h}(x(H-x))$ to the finite element space equipped with a quasi-uniform triangulation given on $[0, H]$. Then we have

$$
\frac{1}{H} \int_{0}^{H} g^{h}(x) d x=O\left(H^{2}\right), \quad\left\|g^{h}(x)\right\|_{H_{00}^{1 / 2}([0, H])}=O\left(H^{2}\right)
$$

for sufficiently small $h$.

Proof. We can obtain these results by a direct calculation for $g$,

$$
\frac{1}{H} \int_{0}^{H} g(x) d x=O\left(H^{2}\right), \quad\|g(x)\|_{H_{00}^{1 / 2}([0, H])}=O\left(H^{2}\right)
$$

and interpolation results for $g^{h}$.

In the BDDC algorithm, we use the Lagrange multiplier function $\psi_{i j}$ across the subdomain interface $F_{i j}=\partial \Omega_{i} \cap \partial \Omega_{j}$ to enforce the primal constraint; see (2.7). We note that $\psi_{i j}$ is the sum of Lagrange multiplier basis functions supported in $\bar{F}_{i j}$. We introduce a subinterval $E_{i j}^{(i)}$ of $F_{i j}$ such that

$$
E_{i j}^{(i)}=\bigcup_{l}\left\{\operatorname{supp}\left(\phi_{l}^{(i)}\right): \operatorname{supp}\left(\phi_{l}^{(i)}\right) \subset \operatorname{supp}\left(\psi_{i j}\right)\right\},
$$

where $\phi_{l}^{(i)}$ are the nodal basis functions in the finite element space $\mathbf{W}_{\Gamma}^{(i)}$. Similarly, we introduce $E_{i j}^{(j)}$ using the nodal basis functions in $\mathbf{W}_{\Gamma}^{(j)}$.

We select such intervals on the boundary of $\Omega_{i}$ and denote them by $\left\{E_{k}\right\}_{k}$ and call them reduced edges of $\Omega_{i}$. We define our edge average as

$$
\bar{v}_{E_{k}}=\frac{\int_{F_{i j}} v \psi_{i j} d s}{\int_{F_{i j}} \psi_{i j} d s}
$$

where $F_{i j}$ is the interface containing $E_{k}$ and $\psi_{i j}$ is the Lagrange multiplier function used for the primal constraint on $F_{i j}$. We use the notation $\bar{v}_{E_{k}}$ for the average value rather than $\bar{v}_{F_{i j}}$ for a simple presentation of the proof in Lemma 4.2.

For a reduced edge $E_{k}=E_{i j}^{(i)} \subset F_{i j} \subset \partial \Omega_{i}$, defined in (4.1), we may consider $E_{k}$ as a straight line with its length $H_{k}\left(\leq H_{i}\right)$. Using Lemma 4.1, we construct such a function $g^{h}$ in the interval $\left[0, H_{k}\right]$ and obtain a function $g_{k}(s)$ defined on $E_{k}$ using an appropriate translation and rotation. We extend $g_{k}(s)$ by zero to $F_{i j}$. For the function $g_{k}$, we can prove

$$
{\overline{g_{k}}}_{E_{k}}=\frac{\int_{F_{i j}} g_{k} \psi_{i j} d s}{\int_{F_{i j}} \psi_{i j} d s}=O\left(H_{k}^{2}\right), \quad\left\|g_{k}\right\|_{H_{00}^{1 / 2}\left(E_{k}\right)}=O\left(H_{k}^{2}\right) ;
$$

Copyright (c) by SIAM. Unauthorized reproduction of this article is prohibited. 


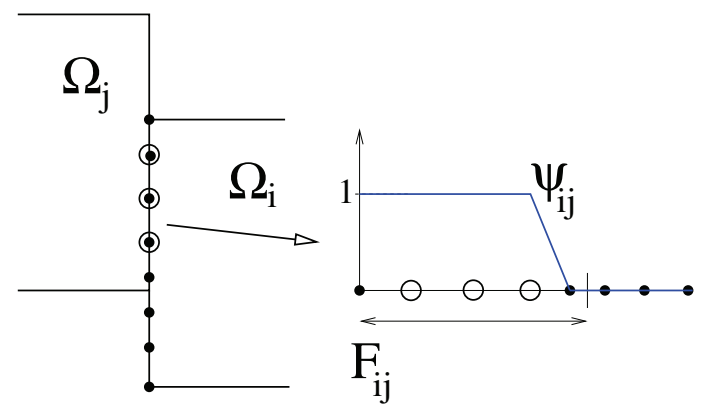

FIG. 2. An example of the function $\psi_{i j}$ with the standard Lagrange multiplier basis in a geometrically nonconforming partition: $\Omega_{i}$ is the nonmortar part of $F_{i j}$, the big white nodes designate the degrees of freedom of Lagrange multiplier basis $\left\{\psi_{i j, k}\right\}_{k}$ supported in $\bar{F}_{i j}$, and $\psi_{i j}=\sum_{k} \psi_{i j, k}$.

see Lemma 4.1. Here $H_{k}$ is the length of $E_{k}$. In the geometrically nonconforming partition, when $F_{i j}$ is a part of the subdomain edge, $\psi_{i j}$ may not be the constant function with the value one on $F_{i j}$; see Figure 2. However, we can see that $\bar{g}_{k_{k}}$ with such $\psi_{i j}$ is similar to the regular average of $g_{k}$ that is used in the conforming finite element case

$$
\overline{g_{k}}=\frac{\int_{F_{i j}} g_{k} d s}{\int_{F_{i j}} 1 d s} .
$$

We note that (4.2) also holds for the case when the length of $H_{k}$ is comparable to the mesh size $h_{i}$. This can be shown by a direct calculation.

LEMma 4.2. Let $\left\{\Omega_{j}^{i}\right\}_{j}$ be the subdomains in a subregion $\Omega^{i}$, and let $\left\{E_{k}\right\}_{k}$ be the reduced edges of $\Omega_{j}^{i}$. For given values $\left\{m_{k}\right\}_{k}$, let $u$ be the minimal energy extension to the subdomain finite element space $V_{i, j}^{h}$ with its average values $\bar{u}_{E_{k}}=m_{k}$ on each $E_{k}$. We then have

$$
C_{1}|u|_{H^{1}\left(\Omega_{j}^{i}\right)}^{2} \leq \sum_{k, l}\left|\bar{u}_{E_{k}}-\bar{u}_{E_{l}}\right|^{2} \leq C_{2}|u|_{H^{1}\left(\Omega_{j}^{i}\right)}^{2}
$$

Proof. We consider a function $v$ in $V_{i, j}^{h}$ defined as

$$
v(x)=\sum_{k} \frac{1}{\overline{g_{k}} E_{k}}\left(\bar{u}_{E_{k}}-\bar{u}_{E_{1}}\right) \phi_{k}(x)+\bar{u}_{E_{1}}
$$

where $\phi_{k}$ is the discrete harmonic extension of $g_{k}$ to $V_{i, j}^{h}$. Here $g_{k}(x)$ is the function which satisfies (4.2) on $E_{k}$ and is zero on $\partial \Omega_{j}^{i} \backslash E_{k}$. We can see easily that

$$
\bar{v}_{E_{k}}=\bar{u}_{E_{k}} .
$$

Since $u$ is the minimal energy extension with the average values $\bar{u}_{E_{k}}=m_{k}$, we have

$$
|u|_{H^{1}\left(\Omega_{j}^{i}\right)}^{2} \leq|v|_{H^{1}\left(\Omega_{j}^{i}\right)}^{2}
$$

Copyright $@$ by SIAM. Unauthorized reproduction of this article is prohibited. 
We consider

$$
\begin{aligned}
|u|_{H^{1}\left(\Omega_{j}^{i}\right)}^{2} & \leq|v|_{H^{1}\left(\Omega_{j}^{i}\right)}^{2} \\
& =\left|\sum_{k} \frac{1}{\overline{g_{k}} E_{k}}\left(\bar{u}_{E_{k}}-\bar{u}_{E_{1}}\right) \phi_{k}(x)+\bar{u}_{E_{1}}\right|_{H^{1}\left(\Omega_{j}^{i}\right)}^{2} \\
& =\left|\sum_{k} \frac{1}{\overline{g_{k}} E_{k}}\left(\bar{u}_{E_{k}}-\bar{u}_{E_{1}}\right) \phi_{k}(x)\right|_{H^{1}\left(\Omega_{j}^{i}\right)}^{2} \\
& \leq C \sum_{k} \frac{1}{\overline{\overline{g k}}_{E_{k}}^{2}}\left(\bar{u}_{E_{k}}-\bar{u}_{E_{1}}\right)^{2}\left|\phi_{k}\right|_{H^{1}\left(\Omega_{j}^{i}\right)}^{2} \\
& \leq C \sum_{k} \frac{1}{{\overline{g_{k}}}_{E_{k}}^{2}}\left(\bar{u}_{E_{k}}-\bar{u}_{E_{1}}\right)^{2}\left\|g_{k}\right\|_{H_{00}^{1 / 2}\left(E_{k}\right)}^{2}
\end{aligned}
$$

where we use [26, Lemma 4.10] or [29, Lemma 2.4] for the last inequality. Applying (4.2) to the above equation, we obtain

$$
|u|_{H^{1}\left(\Omega_{j}^{i}\right)}^{2} \leq C \sum_{k}\left(\bar{u}_{E_{k}}-\bar{u}_{E_{1}}\right)^{2} .
$$

We now prove the other bound as follows:

$$
\begin{aligned}
\sum_{k}\left(\bar{u}_{E_{k}}-\bar{u}_{E_{1}}\right)^{2} & =\sum_{k}\left(\overline{\left(u-\bar{u}_{E_{1}}\right)_{E_{k}}}\right)^{2} \\
& \leq C \sum_{F_{i j} \supset E_{k}, k} \frac{1}{\left(\int_{F_{i j}} \psi_{i j}\right)^{2}}\left\|u-\bar{u}_{E_{1}}\right\|_{L^{2}\left(F_{i j}\right)}^{2}\left\|\psi_{i j}\right\|_{L^{2}\left(F_{i j}\right)}^{2} \\
& \leq C|u|_{H^{1}\left(\Omega_{j}^{i}\right)}^{2} .
\end{aligned}
$$

Here we have used the facts that

$$
\left\|\psi_{i j}\right\|_{L^{2}\left(F_{i j}\right)} \leq C H^{1 / 2}, \quad \int_{F_{i j}} \psi_{i j}=O(H)
$$

the Poincaré inequality

$$
\frac{1}{H}\left\|u-\bar{u}_{E_{1}}\right\|_{L^{2}\left(F_{i j}\right)}^{2} \leq C|u|_{H^{1 / 2}\left(F_{i j}\right)}^{2}
$$

and the trace inequality for the discrete harmonic function $u$

$$
|u|_{H^{1 / 2}\left(F_{i j}\right)}^{2} \leq C|u|_{H^{1}\left(\Omega_{j}^{i}\right)}^{2} .
$$

Here $H$ stands for the diameter of $F_{i j}$.

Since each subregion is a union of subdomains, we might have a subregion with irregular boundaries as in Figure 3. We introduce a new mesh on each subregion $\Omega^{i}$. The purpose of introducing this new mesh is to relate the quadratic form in Lemma 4.2 to one for a conventional finite element space. Here we follow [8, 24].

We construct a triangulation of $\Omega^{i}$ with its node set containing the primal nodes and the subdomain vertices. The vertices of the subdomain $\Omega_{j}^{i}$ are the end points of 

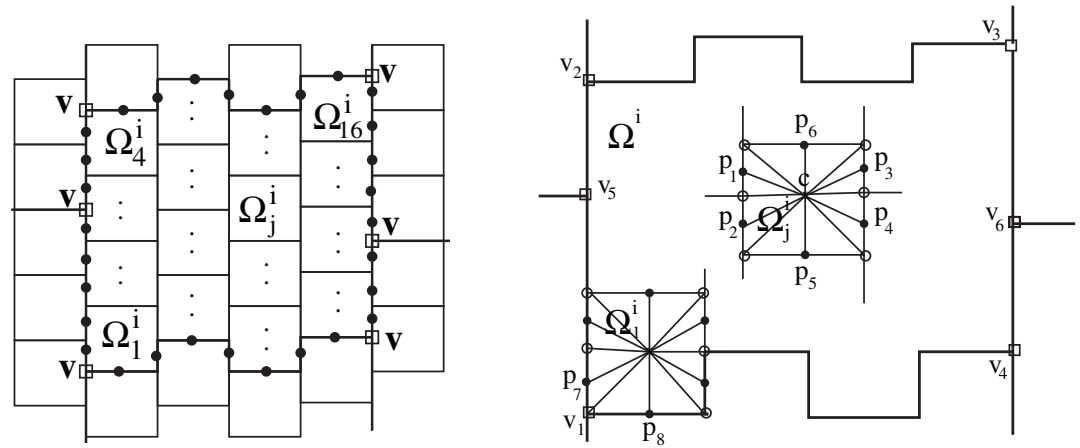

FIG. 3. Left: subregion $\Omega^{i}\left(=\bigcup_{j=1}^{16} \Omega_{j}^{i}\right)$ with irregular boundary; $v$ are subregion vertices, and the nodes at black dots are unknowns at the subregion boundary. Right: a triangulation for the subregion $\Omega^{i} ; p_{k}$ are primal nodes, $c$ is the center of the primal nodes $\left\{p_{k}\right\}_{k=1}^{6}, v_{k}$ are the subregion vertices, and the nodes at white circles are the subdomain vertices.

$F_{j k}=\left(\Omega_{j}^{i} \cap \Omega_{k}^{i}\right)$, where $\Omega_{k}^{i}$ are neighbors of $\Omega_{j}^{i}$. We note that we have one primal unknown for each interface $F_{j k}$. We locate the node corresponding to the primal unknown at the midpoint of the two end points of $F_{j k}$. We call these nodes primal nodes. After introducing the primal nodes in the subdomain $\Omega_{j}^{i}$, we consider the center point of all of these primal nodes; i.e., each component of the center points is the average of each component of the primal nodes. We then connect all primal nodes and vertices to the center point and obtain a triangulation of $\Omega_{j}^{i}$ as in Figure 3 . Finally, the union of such triangulations of $\Omega_{j}^{i}$ gives a triangulation of the subregion $\Omega^{i}$. The corresponding finite element space is denoted by $U_{H}\left(\Omega^{i}\right)$.

We note that the subregion $\Omega^{i}$ is equipped with the triangulation whose nodes consist of the primal nodes, vertices, and the center points of its subdomains $\Omega_{j}^{i}$; see Figure 3. We call the nodes other than the primal nodes the secondary nodes. Among the secondary nodes, we call those at the interior of the subregion $\Omega^{i}$ the interior secondary nodes and those at the boundary of the subregion $\Omega^{i}$ the boundary secondary nodes. In addition, we call two nodes in a triangulation adjacent if they are connected through an edge of the triangulation.

For a function $\phi^{I}(x) \in U_{H}\left(\Omega^{i}\right)$, we define an interpolant $I_{H}^{\Omega^{i}} \phi^{I}(x)$ to $U_{H}\left(\Omega^{i}\right)$ by

$I_{H}^{\Omega^{i}} \phi^{I}(x)=\left\{\begin{array}{l}\phi^{I}(x) \text { if } x \text { is a primal node } I_{H}^{\Omega^{i}} \phi^{I}\left(p_{k}\right)=\phi^{I}\left(p_{k}\right) ; \\ \text { the average of the values at all adjacent primal nodes on edges of } \Omega^{i} \\ \text { if } x \text { is a boundary secondary node } I_{H}^{\Omega^{i}} \phi^{I}\left(v_{1}\right)=\frac{1}{2}\left(\phi^{I}\left(p_{7}\right)+\phi^{I}\left(p_{8}\right)\right) ; \\ \text { the average of the values at all adjacent primal nodes } \\ \text { if } x \text { is an interior secondary node } I_{H}^{\Omega^{i}} \phi^{I}(c)=\frac{1}{6} \sum_{k=1}^{6} \phi^{I}\left(p_{k}\right) .\end{array}\right.$

Here we presented the specific values of $I_{H}^{\Omega^{i}} \phi^{I}(x)$ for the case in Figure 3.

We recall that $\mathbf{W}_{c}^{(i)}$ is the discrete space of values at the primal nodes in the subregion $\Omega^{i}$ and $\mathbf{W}_{\Gamma_{c}}^{(i)}$ is its trace space on the subregion boundary. All of these nodes correspond to the primal unknowns of the subdomain partition. Given any $\phi \in \mathbf{W}_{c}^{(i)}$, we can find a function $\phi^{I} \in U_{H}\left(\Omega^{i}\right)$ with the values at the primal nodes equal to the components of $\phi$ that correspond to the primal unknowns associated with 
those nodes. For such $\phi \in \mathbf{W}_{c}^{(i)}$, we define a similar interpolant to $U_{H}\left(\Omega^{i}\right)$ by

$$
I_{H}^{\Omega^{i}} \phi:=I_{H}^{\Omega^{i}} \phi^{I}(x) .
$$

We note that the function $\phi^{I}$ is not unique, but $I_{H}^{\Omega^{i}} \phi(x)$ will be determined uniquely since the interpolation $I_{H}^{\Omega^{i}}$ depends only on the values at the primal nodes.

We now define a mapping $I_{H}^{\partial \Omega^{i}} \phi$ from $\mathbf{W}_{\Gamma_{c}}^{(i)}$ to the space $U_{H}\left(\partial \Omega^{i}\right)$, the trace space of $U_{H}\left(\Omega^{i}\right)$, by

$$
I_{H}^{\partial \Omega^{i}} \phi=\left.\left(I_{H}^{\Omega^{i}} \phi_{e}\right)\right|_{\partial \Omega^{i}} .
$$

Here $\phi_{e}$ is any function in $\mathbf{W}_{c}^{(i)}$ such that $\left.\phi_{e}\right|_{\partial \Omega^{i}}=\phi$. The map is well defined, since the values of $I_{H}^{\Omega^{i}} \phi_{e}$ on the subregion boundary depend only on the values of $\phi_{e}$ at the primal nodes on the subregion boundary.

We introduce the range spaces $I_{H}^{\Omega^{i}}\left(\mathbf{W}_{c}^{(i)}\right)$ and $I_{H}^{\partial \Omega^{i}}\left(\mathbf{W}_{\Gamma_{c}}^{(i)}\right)$ and denote them, respectively, by

$$
S_{H}\left(\Omega^{i}\right):=I_{H}^{\Omega^{i}}\left(\mathbf{W}_{c}^{(i)}\right) \text { and } S_{H}\left(\partial \Omega^{i}\right):=I_{H}^{\partial \Omega^{i}}\left(\mathbf{W}_{\Gamma_{c}}^{(i)}\right) .
$$

We note that $S_{H}\left(\Omega^{i}\right)$ and $S_{H}\left(\partial \Omega^{i}\right)$ are the subspaces of $U_{H}\left(\Omega^{i}\right)$ and $U_{H}\left(\partial \Omega^{i}\right)$, respectively.

In order to prove Lemma 4.5, which plays an important role in our condition number estimate, we need to establish the equivalence between the $H^{1}$-norm of the discrete harmonic extensions in the spaces $S_{H}\left(\Omega^{i}\right)$ and $U_{H}\left(\Omega^{i}\right)$ for any $\phi \in S_{H}\left(\partial \Omega^{i}\right)$.

LEMma 4.3. There exists a constant $C>0$, independent of $H$ and $\left|\Omega^{i}\right|$, the volume of $\Omega^{i}$, but dependent on the shape regularity of the triangulation of $\Omega^{i}$, such that

$\left|I_{H}^{\Omega^{i}} \phi\right|_{H^{1}\left(\Omega^{i}\right)} \leq C|\phi|_{H^{1}\left(\Omega^{i}\right)} \quad$ and $\quad\left\|I_{H}^{\Omega^{i}} \phi\right\|_{L^{2}\left(\Omega^{i}\right)} \leq C\|\phi\|_{L^{2}\left(\Omega^{i}\right)} \quad \forall \phi \in U_{H}\left(\Omega^{i}\right)$.

Proof. See [8, Lemma 6.1].

Lemma 4.4. For $\phi \in S_{H}\left(\partial \Omega^{i}\right)$,

$$
\inf _{v \in S_{H}\left(\Omega^{i}\right),\left.v\right|_{\partial \Omega^{i}}=\phi}\|v\|_{H^{1}\left(\Omega^{i}\right)} \approx \inf _{v \in U_{H}\left(\Omega^{i}\right),\left.v\right|_{\partial \Omega^{i}}=\phi}\|v\|_{H^{1}\left(\Omega^{i}\right)}
$$

and

$$
\inf _{v \in S_{H}\left(\Omega^{i}\right),\left.v\right|_{\partial \Omega^{i}}=\phi}|v|_{H^{1}\left(\Omega^{i}\right)} \approx \inf _{v \in U_{H}\left(\Omega^{i}\right),\left.v\right|_{\partial \Omega^{i}}=\phi}|v|_{H^{1}\left(\Omega^{i}\right)} .
$$

Here $S_{H}\left(\Omega^{i}\right)$ is a subspace of $U_{H}\left(\Omega^{i}\right)$.

Proof. For the first equivalence, since $S_{H}\left(\Omega^{i}\right)$ is a subspace of $U_{H}\left(\Omega^{i}\right)$, we need only to prove that

$$
\inf _{v \in S_{H}\left(\Omega^{i}\right),\left.v\right|_{\partial \Omega^{i}}=\phi}\|v\|_{H^{1}\left(\Omega^{i}\right)} \leq C \inf _{v \in U_{H}\left(\Omega^{i}\right),\left.v\right|_{\partial \Omega^{i}}=\phi}\|v\|_{H^{1}\left(\Omega^{i}\right)} .
$$

Given any function $v \in U_{H}\left(\Omega^{i}\right)$ with $v=\phi$ on $\partial \Omega^{i}$, let $w=I_{H}^{\Omega^{i}} v \in S_{H}\left(\Omega^{i}\right)$. Since $\phi \in S_{H}\left(\partial \Omega^{i}\right)$ and by the definitions of $I_{H}^{\Omega^{i}}$ and $I_{H}^{\partial \Omega^{i}}$, we have $w=\phi$ on $\partial \Omega^{i}$. 
Moreover, by Lemma 4.3 , we have $\|w\|_{H^{1}\left(\Omega^{i}\right)}=\left\|I_{H}^{\Omega^{i}} v\right\|_{H^{1}\left(\Omega^{i}\right)} \leq C\|v\|_{H^{1}\left(\Omega^{i}\right)}$ for any $v \in U_{H}\left(\Omega^{i}\right)$ with $v=\phi$ on $\partial \Omega^{i}$, and we proved the first equivalence. The second equivalence can be obtained similarly.

We note that the hidden constants in the equivalences in Lemma 4.4 depend on the shape regularity of the partition of the subregion $\Omega^{i}$ by the subdomains $\Omega_{j}^{i}$. The constants in the following Lemmas 4.5, 4.7, 4.8, and 5.1 and Theorem 5.2 will have the same dependence. For a discussion of the shape regularity of a partition, see [5].

Lemma 4.5. There exist constants $C_{1}$ and $C_{2}>0$, independent of $\widehat{H}, H, h$, and $\rho_{i}$, such that for all $w_{i} \in \mathbf{W}_{\Gamma_{c}}^{(i)}$,

$$
\begin{aligned}
& C_{1} \rho_{i} \inf _{v \in U_{H}\left(\Omega^{i}\right),\left.v\right|_{\partial \Omega^{i}=I_{H}^{\partial \Omega^{i}}} w_{i}}|v|_{H^{1}\left(\Omega^{i}\right)}^{2} \leq\left\langle T^{(i)} w_{i}, w_{i}\right\rangle \leq C_{2} \rho_{i} \inf _{v \in U_{H}\left(\Omega^{i}\right),\left.v\right|_{\partial \Omega^{i}=I_{H}^{\partial \Omega^{i}} w_{i}}}|v|_{H^{1}\left(\Omega^{i}\right)}^{2}, \\
& \text { where }\left\langle T^{(i)} w_{i}, w_{i}\right\rangle=w_{i}^{T} T^{(i)} w_{i}=\left|w_{i}\right|_{T^{(i)}}^{2} \text { and } T^{(i)}=S_{\Pi_{\Gamma_{c} \Gamma_{c}}}^{(i)}-S_{\Pi_{\Gamma_{c} I_{c}}}^{(i)}\left(S_{\Pi_{I_{c} I_{c}}}^{(i)}\right)^{-1}\left(S_{\Pi_{\Gamma_{c} I_{c}}}^{(i)}\right)^{T} .
\end{aligned}
$$

Proof. By the definition of $T^{(i)}$, we have

$$
\begin{aligned}
& \left\langle T^{(i)} w_{i}, w_{i}\right\rangle=\inf _{v \in \mathbf{W}_{c}^{(i)},\left.v\right|_{\partial \Omega^{i}}=w_{i}}|v|_{S_{\Pi}^{(i)}}^{2} \\
= & \inf _{v \in \mathbf{W}_{c}^{(i)},\left.v\right|_{\partial \Omega^{i}=w_{i}}} \rho_{i} \sum_{j=1}^{N_{i}}\left(\inf _{u \in V_{i, j}^{h}, u_{E_{l}}=v_{l}, E_{l} \subset \partial \Omega_{j}^{i}}|u|_{H^{1}\left(\Omega_{j}^{i}\right)}^{2}\right) \\
\approx & \inf _{v \in \mathbf{W}_{c}^{(i)},\left.v\right|_{\partial \Omega^{i}}=w_{i}} \rho_{i} \sum_{j=1}^{N_{i}} \sum_{k_{1}, k_{2}}\left|v_{k_{1}}-v_{k_{2}}\right|^{2} \\
\approx & \inf _{v \in \mathbf{W}_{c}^{(i)},\left.v\right|_{\partial \Omega^{i}=w_{i}}} \rho_{i}\left|I_{H}^{\Omega^{i}} v\right|_{H^{1}\left(\Omega^{i}\right)}^{2}=\inf _{v \in S_{H}\left(\Omega^{i}\right),\left.v\right|_{\partial \Omega^{i}}=I_{H}^{\partial \Omega^{i} w_{i}}} \rho_{i}|v|_{H^{1}\left(\Omega^{i}\right)}^{2} \\
\approx & \inf _{v \in U_{H}\left(\Omega^{i}\right),\left.v\right|_{\partial \Omega^{i}}=I_{H}^{\partial \Omega^{i}} w_{i}} \rho_{i}|v|_{H^{1}\left(\Omega^{i}\right)}^{2} .
\end{aligned}
$$

We use Lemma 4.2 for the third bound, the definitions of $I_{H}^{\Omega^{i}}$ and $I_{H}^{\partial \Omega^{i}}$ for the fourth and fifth bounds, and Lemma 4.4 for the final one. Here $v_{l}$ stands for the value of $v \in \mathbf{W}_{c}^{(i)}$ at the primal node corresponding to the reduced edge $E_{l}$ of the subdomain $\Omega_{j}^{i}$.

Next we refer to Lemma 4.2 in [15] for subdomains with irregular boundary. We rewrite this lemma for our subregions with irregular boundary.

Lemma 4.6. Let $F^{i j}$ be an edge common to the boundaries of $\Omega^{i}$ and $\Omega^{j}$. For all $w_{i} \in U_{H}\left(\Omega^{i}\right)$ and $w_{j} \in U_{H}\left(\Omega^{j}\right)$, which have the same edge average over the common edge $F^{i j}$, we have

$$
\begin{aligned}
\left|\mathcal{H}^{i}\left(\vartheta_{F^{i j}}\left(w_{i}-w_{j}\right)\right)\right|_{H^{1}\left(\Omega^{i}\right)}^{2} \leq & C\left(1+\log \left(\widehat{H}_{i} / H_{i}\right)\right)^{2}\left|w_{i}\right|_{H^{1}\left(\Omega^{i}\right)}^{2} \\
& +C\left(1+\log \left(\widehat{H}_{j} / H_{j}\right)\right)^{2}\left|w_{j}\right|_{H^{1}\left(\Omega^{j}\right)}^{2}
\end{aligned}
$$

where $\vartheta_{F^{i j}}$ is the discrete harmonic extension of $I_{H}^{\partial \Omega^{i}}\left(\zeta_{F^{i j}}\right)$ to $U_{H}\left(\Omega^{i}\right)$ and $\zeta_{F^{i j}}$ has its value one at the nodes in $F^{i j}$ and zero at the other part. Here $\widehat{H}_{i}$ and $\widehat{H}_{j}$ are subregion diameters, and $H_{i}$ and $H_{j}$ are the element sizes of finite element spaces $U_{H}\left(\Omega^{i}\right)$ and $U_{H}\left(\Omega^{j}\right)$, respectively. In addition, $\mathcal{H}^{i}(v)$ denotes the discrete harmonic extension of $v$ restricted on the boundary of $\Omega^{i}$ to $U_{H}\left(\Omega^{i}\right)$.

Copyright (c) by SIAM. Unauthorized reproduction of this article is prohibited. 
We define the interface average operator $E_{D_{c}}$ on $\widetilde{\mathbf{W}}_{\Gamma_{c}}$ as $E_{D_{c}}=\widetilde{R}_{\Gamma_{c}} \widetilde{R}_{D_{c}, \Gamma_{c}}^{T}$, which computes the averages across the subregion interface $\Gamma_{c}$ and then distributes the averages to the unknowns at the subregion boundaries. The interface average operator $E_{D_{c}}$ has the following property.

LEMMA 4.7.

$$
\left|E_{D_{c}} \mathbf{w}_{\Gamma_{c}}\right|_{\widetilde{T}}^{2} \leq C\left(1+\log \frac{\widehat{H}}{H}\right)^{2}\left|\mathbf{w}_{\Gamma_{c}}\right|_{\widetilde{T}}^{2}
$$

for any $\mathbf{w}_{\Gamma_{c}} \in \widetilde{\mathbf{W}}_{\Gamma_{c}}$, where $C$ is a positive constant independent of $\widehat{H}, H, h$, and the coefficients of (2.1), and $\widetilde{T}$ is defined in (3.7).

Proof. We can follow the proof of [30, Lemma 5]. Given any $\mathbf{w}_{\Gamma_{c}} \in \widetilde{\mathbf{W}}_{\Gamma_{c}}$, we have

$$
\begin{aligned}
& \left|E_{D_{c}} \mathbf{w}_{\Gamma_{c}}\right|_{\widetilde{T}}^{2} \\
\leq & 2\left(\left|\mathbf{w}_{\Gamma_{c}}\right|_{\widetilde{T}}^{2}+\left|\mathbf{w}_{\Gamma_{c}}-E_{D_{c}} \mathbf{w}_{\Gamma_{c}}\right|_{\widetilde{T}}^{2}\right) \\
\leq & 2\left(\left|\mathbf{w}_{\Gamma_{c}}\right|_{\widetilde{T}}^{2}+\left|R_{\Gamma_{c}}\left(\mathbf{w}_{\Gamma_{c}}-E_{D_{c}} \mathbf{w}_{\Gamma_{c}}\right)\right|_{T}^{2}\right) \\
= & 2\left(\left|\mathbf{w}_{\Gamma_{c}}\right|_{\widetilde{T}}^{2}+\sum_{i=1}^{N_{c}}\left|\left(\mathbf{w}_{\Gamma_{c}}-E_{D_{c}} \mathbf{w}_{\Gamma_{c}}\right)_{i}\right|_{T^{(i)}}^{2}\right),
\end{aligned}
$$

where $\left(\mathbf{w}_{\Gamma_{c}}-E_{D_{c}} \mathbf{w}_{\Gamma_{c}}\right)_{i}$ is the restriction of $\mathbf{w}_{\Gamma_{c}}-E_{D_{c}} \mathbf{w}_{\Gamma_{c}}$ to the subregion $\Omega^{i}$. Also let $w_{i}$ be the restriction of $\mathbf{w}_{\Gamma_{c}}$ to the subregion $\Omega^{i}$ and set

$$
v_{i}(x):=\left(\mathbf{w}_{\Gamma_{c}}-E_{D_{c}} \mathbf{w}_{\Gamma_{c}}\right)_{i}(x)=\sum_{j \in \mathcal{N}_{x}} \delta_{c, j}^{\dagger}\left(w_{i}(x)-w_{j}(x)\right), \quad x \in \partial \Omega^{i} \cap \Gamma_{c} .
$$

Here $\mathcal{N}_{x}$ is the set of indices of the subregions that have $x$ on their boundaries. We recall the definition for $\delta_{c, j}^{\dagger}$ in (3.10). It satisfies

$$
\rho_{i}\left(\delta_{c, j}^{\dagger}\right)^{2} \leq \min \left(\rho_{i}, \rho_{j}\right)
$$

Let $\zeta_{F}$ be unknowns in $\mathbf{W}_{\Gamma_{c}}^{(i)}$ with its values one at the nodes in $F$ and zero at the other nodes. We also need a function in the space $U_{H}\left(\Omega^{i}\right)$, denoted by $\vartheta_{F}$, which is the discrete harmonic extension of $I_{H}^{\partial \Omega^{i}}\left(\zeta_{F}\right)$ to $U_{H}\left(\Omega^{i}\right)$. We note that $x$ in (4.6) are from the subdomain primal unknowns; they belong to exactly two subregions as in Figure 3 so that we have

$$
\left|v_{i}\right|_{T^{(i)}}^{2} \leq C \sum_{F^{i j} \subset \partial \Omega^{i}}\left|\zeta_{F^{i j}} v_{i}\right|_{T^{(i)}}^{2}
$$

where $F^{i j}$ is the common interface of the subregions $\Omega^{i}$ and $\Omega^{j}$. 
We then obtain

$$
\begin{aligned}
& \left|\zeta_{F^{i j}} v_{i}\right|_{T^{(i)}}^{2} \inf _{\leq} C \rho_{i \in U_{H}\left(\Omega^{i}\right),\left.v\right|_{\partial \Omega^{i}}=I_{H}^{\partial \Omega^{i}}\left(\zeta_{F^{i j}} v_{i}\right)}|v|_{H^{1}\left(\Omega^{i}\right)}^{2} \\
= & C \rho_{i} \delta_{c, j}^{\dagger^{2}}\left|\mathcal{H}^{i}\left(I_{H}^{\partial \Omega^{i}}\left(\zeta_{F_{i j}}\left(w_{i}-w_{j}\right)\right)\right)\right|_{H^{1}\left(\Omega^{i}\right)}^{2} \\
= & C \rho_{i} \delta_{c, j}^{\dagger^{2}}\left|\mathcal{H}^{i}\left(I_{H}^{\partial \Omega^{i}}\left(\zeta_{F_{i j}}\left(I_{H}^{\partial \Omega^{i}}\left(w_{i}\right)-I_{H}^{\partial \Omega^{j}}\left(w_{j}\right)\right)\right)\right)\right|_{H^{1}\left(\Omega^{i}\right)}^{2} \\
\leq & C \rho_{i} \delta_{c, j}^{\dagger^{2}} \mid I_{H}^{\Omega^{i}}\left(\left.\mathcal{H}^{i}\left(\vartheta_{F_{i j}}\left(\mathcal{H}^{i}\left(I_{H}^{\partial \Omega^{i}}\left(w_{i}\right)\right)-\mathcal{H}^{j}\left(I_{H}^{\partial \Omega^{j}}\left(w_{j}\right)\right)\right)\right)\right|_{H^{1}\left(\Omega^{i}\right)} ^{2}\right. \\
\leq & C \rho_{i} \delta_{c, j}^{\dagger^{2}}\left|\mathcal{H}^{i}\left(\vartheta_{F_{i j}}\left(\mathcal{H}^{i}\left(I_{H}^{\partial \Omega^{i}}\left(w_{i}\right)\right)-\mathcal{H}^{j}\left(I_{H}^{\partial \Omega^{j}}\left(w_{j}\right)\right)\right)\right)\right|_{H^{1}\left(\Omega^{i}\right)}^{2} .
\end{aligned}
$$

Here $\mathcal{H}^{i}(v)$ is the discrete harmonic extension of $v$ restricted on the boundary of $\Omega^{i}$ to $U_{H}\left(\Omega^{i}\right)$, and Lemmas 4.5 and 4.3 are used for the first and last inequalities, respectively.

We can estimate the term in (4.9) by Lemma 4.6 to obtain

$$
\left|\zeta_{F^{i j}} v_{i}\right|_{T^{(i)}}^{2} \leq C \rho_{i} \delta_{c, j}^{+2}\left(1+\log \frac{\widehat{H}}{H}\right)^{2} \sum_{k=i, j}\left|\mathcal{H}^{k}\left(I_{H}^{\partial \Omega^{k}}\left(w_{k}\right)\right)\right|_{H^{1}\left(\Omega^{k}\right)}^{2},
$$

where $w_{i}$ and $w_{j}$ have the same edge average on $F^{i j}$.

Combining the above inequality with (4.7) and Lemma 4.5, we obtain

$$
\left|\zeta_{F^{i j}} v_{i}\right|_{T^{(i)}}^{2} \leq C\left(1+\log \frac{\widehat{H}}{H}\right)^{2}\left(\left|w_{i}\right|_{T^{(i)}}^{2}+\left|w_{j}\right|_{T^{(j)}}^{2}\right) .
$$

From (4.5), (4.6), (4.8), and the above inequality, the desired bound then follows:

$$
\left|E_{D_{c}} \mathbf{w}_{\Gamma_{c}}\right|_{\widetilde{T}}^{2} \leq C\left(1+\log \frac{\widehat{H}}{H}\right)^{2}\left|\mathbf{w}_{\Gamma_{c}}\right|_{\widetilde{T}}^{2} .
$$

Using Lemma 4.7, we can prove the following result; see [32, Lemma 4.6] or [31, Lemma 4.7].

Lemma 4.8. Given any $\mathbf{u}_{\boldsymbol{\Gamma}} \in \widehat{\mathbf{W}}_{\Gamma}$, let $\boldsymbol{\Psi}=\Phi^{T} \widetilde{R}_{D, \Gamma} \mathbf{u}_{\Gamma}$. We have

$$
\boldsymbol{\Psi}^{T} S_{\Pi}^{-1} \boldsymbol{\Psi} \leq \boldsymbol{\Psi}^{T} M_{\Pi}^{-1} \boldsymbol{\Psi} \leq C\left(1+\log \frac{\widehat{H}}{H}\right)^{2} \boldsymbol{\Psi}^{T} S_{\Pi}^{-1} \boldsymbol{\Psi} .
$$

5. Condition number estimate for the new preconditioner. In order to estimate the condition number for the system with the new preconditioner $\widetilde{M}^{-1}$, we compare it to the system with the preconditioner $M^{-1}$ by using Lemma 4.8.

Lemma 5.1. Given any $\mathbf{u}_{\boldsymbol{\Gamma}} \in \widehat{\mathbf{W}}_{\Gamma}$,

$$
\mathbf{u}_{\Gamma}^{T} M^{-1} \mathbf{u}_{\Gamma} \leq \mathbf{u}_{\Gamma}^{T} \widetilde{M}^{-1} \mathbf{u}_{\Gamma} \leq C\left(1+\log \frac{\widehat{H}}{H}\right)^{2} \mathbf{u}_{\Gamma}^{T} M^{-1} \mathbf{u}_{\Gamma}
$$

Proof. See [32, Lemma 5.1] or [31, Lemma 5.1]. 
THEOREM 5.2. The condition number for the system with the three-level preconditioner $\widetilde{M}^{-1}$ is bounded by $C(1+\log (\widehat{H} / H))^{2}(1+\log (H / h))^{2}$.

Proof. Combining the condition number bound in (2.16) for the two-level BDDC method and Lemma 5.1, we find that the condition number for the three-level method is bounded by $C(1+\log (\widehat{H} / H))^{2}(1+\log (H / h))^{2}$.

6. Numerical experiments. In this section, we present numerical results for the suggested algorithm. We consider the elliptic problem in the unit rectangular domain $\Omega=[0,1]^{2}$ :

$$
\begin{aligned}
-\nabla \cdot(\rho(x, y) \nabla u(x, y)) & =f(x, y), \quad(x, y) \in \Omega, \\
u(x, y) & =0, \quad(x, y) \in \partial \Omega,
\end{aligned}
$$

where $f(x, y)$ is given in $L^{2}(\Omega)$. In our experiments, we performed the conjugate gradient iterations up to the relative residual norm reduced by a factor of $10^{6}$.

We test our algorithm by two sets of the numerical experiments. In the first set of the experiments, we take $\rho(x, y)=1$ everywhere in the domain. While in the second set of the experiments, we take $\rho(x, y)$ to be constants in each subregion but to have large jumps across the subregion boundaries. In each experiment set, we performed the computations for both geometrically conforming and nonconforming subdomain partitions and used the Lagrange multiplier space with dual basis. All of these numerical results are consistent with our theory.

The geometrically conforming partitions are obtained from the uniform rectangles of length $1 / N$, where $N$ denotes the number of subdomains in each $x$ and $y$ directional edge of $\Omega$. For a given $N$, we obtain $N^{2}$ uniform rectangular subdomains. Each subdomain is equipped with finite elements that can be nonmatching across the subdomain interface. In the three-level algorithm, we group subdomains to obtain a uniform rectangular subregion partition. Each subregion has $\widehat{N}$ subdomains in its $x$ and $y$-directional edges.

To obtain a geometrically nonconforming subdomain partition, we first partition $\Omega$ into $N$ uniformly vertical strips in the $x$-direction and then divide each strip into $N$ or $N+1$ rectangles successively. We group subdomains to obtain a subregion partition with $\widehat{N}=\widehat{H} / H$, the number of subdomains across an edge of a subregion. Figure 4 shows a geometrically conforming subdomain partition, a geometrically nonconforming subdomain partition, and their subregion partitions when $N=16$ and $\widehat{N}=4$.

In the first set of the experiments, we set $\rho(x, y)=1$. We perform the exact two-level BDDC algorithm and the inexact three-level BDDC algorithm to see the scalability in terms of the number of subdomains and the number of subregions, respectively. Tables 1 and 2 show the condition numbers and the number of iterations in geometrically conforming and nonconforming partitions, respectively. Here $N_{d}$ and $N_{c}$ denote the number of subdomains and the number of subregions, respectively. In the inexact case, the subdomain problem size and the subregion problem size are fixed, and in the exact case the subdomain problem size is fixed. Both cases show a good scalability. In Tables 1 and 2, each row corresponds to the same subdomain partition, i.e., the same coarse problem $S_{\Pi}$ in (2.15). The inexact case solves the coarse problem approximately by applying a BDDC preconditioner to solve the coarse problem $S_{\Pi}$. We can observe that, when using the inexact coarse problem, there are only slight increases in the condition numbers and the number of iterations compared to the exact coarse problem. However, the coarse problem is solved quite cheaply in the inexact case.

Copyright $@$ by SIAM. Unauthorized reproduction of this article is prohibited. 

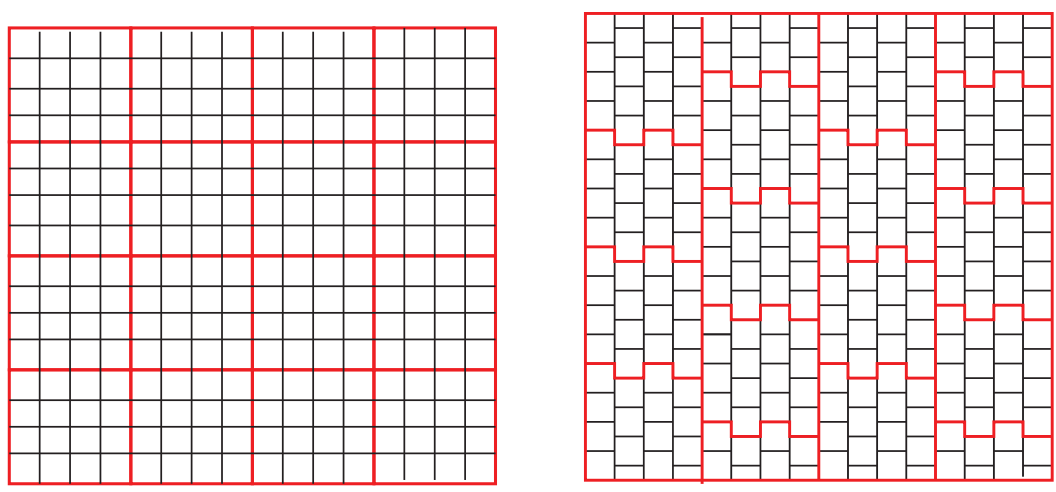

FIG. 4. Examples of subdomain and subregion partitions: smaller rectangles are subdomains and each subregion (with thick boundary) is a group of subdomains. Left: a geometrically conforming subdomain partition of $16^{2}$ subdomains $(N=16)$ and its subregion partition with 4 subdomains $(\widehat{N}=$ 4) across each subregion (the number of subregions are $4^{2}$ ). Right: a geometrically nonconforming subdomain partition of $16^{2}+8$ subdomains $(N=16)$ and its subregion partition with 4 subdomains $(\widehat{N}=4)$ across each subregion (the number of subregions are $\left.4^{2}+2\right)$.

TABLE 1

Geometrically conforming subdomain partitions. Left three columns: scalability as the increase of the number of subdomains $N_{d}$ for the BDDC algorithm with the exact coarse problem when the subdomain problem sizes are fixed with $(H / h)=5$ or 4 . Right three columns: scalability as the increase of the number of subregions $N_{c}$ for the BDDC algorithm with an inexact coarse problem when the subregion problem sizes, $\widehat{N}=(\widehat{H} / H)=4$, and the subdomain problem sizes, $(H / h)=5$ or 4 , are fixed.

\begin{tabular}{|c||c|c||c||c|c|}
\hline \multicolumn{3}{|c||}{ Exact } & \multicolumn{3}{c|}{ Inexact } \\
\hline$N_{d}$ & Cond & Iter & $N_{c}$ & Cond & Iter \\
\hline $16^{2}$ & 9.18 & 18 & $4^{2}$ & 9.67 & 19 \\
$32^{2}$ & 9.26 & 17 & $8^{2}$ & 10.11 & 21 \\
$64^{2}$ & 9.28 & 17 & $16^{2}$ & 10.13 & 20 \\
$80^{2}$ & 9.29 & 17 & $20^{2}$ & 10.13 & 20 \\
\hline
\end{tabular}

TABLE 2

Geometrically nonconforming partitions. Left three columns: scalability as the increase of the number of subdomains $N_{d}$ for the BDDC algorithm with the exact coarse problem when the subdomain problem sizes are fixed with $(H / h)=6,8$, or 10 . Right three columns: scalability as the increase of the number of subregions $N_{c}$ for the BDDC algorithm with an inexact coarse problem when the subregion problem sizes, $\widehat{N}=(\widehat{H} / H)=4$, and the subdomain problem sizes, $(H / h)=6,8$, or 10 , are fixed.

\begin{tabular}{|c||c|c||c||c|c|}
\hline \multicolumn{3}{|c||}{ Exact } & \multicolumn{3}{c|}{ Inexact } \\
\hline$N_{d}$ & Cond & Iter & $N_{c}$ & Cond & Iter \\
\hline $16^{2}+8$ & 12.36 & 23 & $4^{2}+2$ & 12.70 & 26 \\
$32^{2}+16$ & 12.37 & 24 & $8^{2}+4$ & 12.79 & 27 \\
$64^{2}+32$ & 12.40 & 24 & $16^{2}+8$ & 12.81 & 29 \\
$80^{2}+40$ & 12.41 & 25 & $20^{2}+10$ & 12.82 & 29 \\
\hline
\end{tabular}

Tables 3 and 4 present the results of the three-level algorithm by changing the subregion problem size and the subdomain problem size. Tables 3 and 4 are for geometrically conforming and nonconforming subdomain partitions, respectively. Both results are consistent with our theory. 
TABLE 3

Geometrically conforming subdomain partitions with $4 \times 4$ subregions. Left three columns: scalability as the increase of the subregion problem size $\widehat{N}$ when the subdomain problem sizes are fixed with $n=(H / h)=5$ or 6 . Right three columns: scalability as the increase of the subdomain problem size $n$ when the subregion problem sizes are fixed with $\widehat{N}=(\widehat{H} / H)=4$.

\begin{tabular}{|c||c|c||c||c|c|}
\hline \multicolumn{2}{|c||}{$4 \times 4$ subregions, $n$ fixed } & \multicolumn{3}{c|}{$4 \times 4$ subregions, $\widehat{N}$ fixed } \\
\hline$\widehat{N}=\frac{\widehat{H}}{H}$ & Cond & Iter & $n=\frac{H}{h}$ & Cond & Iter \\
\hline 4 & 9.67 & 19 & $(5,4)$ & 9.67 & 19 \\
8 & 10.57 & 20 & $(10,8)$ & 13.23 & 23 \\
16 & 11.73 & 24 & $(20,16)$ & 17.20 & 26 \\
20 & 12.16 & 25 & $(25,20)$ & 18.56 & 26 \\
\hline
\end{tabular}

TABLE 4

Geometrically nonconforming subdomain partitions with $4^{2}+2$ subregions. Left three columns: scalability as the increase of the subregion problem size $\widehat{N}$ when the subdomain problem sizes are fixed with $n=(H / h)=6,8$, or 10 . Right three columns: scalability as the increase of the subdomain problem size $n$ when the subregion problem sizes are fixed with $\widehat{N}=(\widehat{H} / H)=4$.

\begin{tabular}{|c||c|c||c||c|c|}
\hline \multicolumn{2}{|c||}{$4^{2}+2$ subregions, $n$ fixed } & \multicolumn{3}{c|}{$4^{2}+2$ subregions, $\widehat{N}$ fixed } \\
\hline$\widehat{N}=\frac{\widehat{H}}{H}$ & Cond & Iter & $n=\frac{H}{h}$ & Cond & Iter \\
\hline 4 & 12.70 & 26 & $(6,8,10)$ & 12.70 & 26 \\
8 & 13.11 & 28 & $(8,10,12)$ & 14.12 & 27 \\
16 & 13.77 & 29 & $(18,20,22)$ & 18.39 & 30 \\
20 & 14.01 & 30 & $(24,26,28)$ & 20.05 & 30 \\
\hline
\end{tabular}

TABLE 5

Discontinuous coefficient case for geometrically conforming subdomain partitions. Left three columns: scalability as the increase of the number of subdomains $N_{d}$ for the BDDC algorithm with the exact coarse problem when the subdomain problem sizes are fixed with $(H / h)=5$ or 4 . Right three columns: scalability as the increase of the number of subregions $N_{c}$ for the BDDC algorithm with an inexact coarse problem when the subregion problem sizes, $\widehat{N}=(\widehat{H} / H)=4$, and the subdomain problem sizes, $(H / h)=5$ or 4 , are fixed.

\begin{tabular}{|c||c|c||c||c|c|}
\hline \multicolumn{3}{|c||}{ Exact } & \multicolumn{3}{c|}{ Inexact } \\
\hline$N_{d}$ & Cond & Iter & $N_{c}$ & Cond & Iter \\
\hline $16^{2}$ & 9.18 & 19 & $4^{2}$ & 9.55 & 22 \\
$32^{2}$ & 9.22 & 19 & $8^{2}$ & 10.01 & 23 \\
$64^{2}$ & 9.27 & 18 & $16^{2}$ & 10.17 & 22 \\
$80^{2}$ & 9.27 & 18 & $20^{2}$ & 10.19 & 21 \\
\hline
\end{tabular}

In our second set of the numerical experiments, we test our algorithm with discontinuous coefficients $\rho(x, y)$. The values $\rho(x, y)$ are selected among $1,10,100$, and 1000. They are constants in each subregion, but they can have jump across subregion boundaries.

As before, we compare the two-level and the three-level algorithms with the same coarse problem size on the geometrically conforming and nonconforming subdomain partitions. The results are reported in Tables 5 and 6 . The three-level algorithm gives slightly more iterations due to solving the coarse problem inexactly. However, the computation cost is reduced for each iteration resulting in a faster computing time than the two-level algorithm.

Tables 7 and 8 show the number of iterations and condition numbers of the threelevel algorithm regarding the subregion problem size and the subdomain problem 
TABLE 6

Discontinuous coefficient case for geometrically nonconforming partitions. Left three columns: scalability as the increase of the number of subdomains $N_{d}$ for the BDDC algorithm with the exact coarse problem when the subdomain problem sizes are fixed with $(H / h)=6,8$, or 10 . Right three columns: scalability as the increase of the number of subregions $N_{c}$ for the BDDC algorithm with an inexact coarse problem when the subregion problem sizes, $\widehat{N}=(\widehat{H} / H)=4$, and the subdomain problem sizes, $(H / h)=6,8$, or 10 , are fixed.

\begin{tabular}{|c||c|c||c||c|c|}
\hline \multicolumn{3}{|c||}{ Exact } & \multicolumn{3}{c|}{ Inexact } \\
\hline$N_{d}$ & Cond & Iter & $N_{c}$ & Cond & Iter \\
\hline $16^{2}+8$ & 11.85 & 25 & $4^{2}+2$ & 11.87 & 27 \\
$32^{2}+16$ & 12.25 & 26 & $8^{2}+4$ & 12.56 & 29 \\
$64^{2}+32$ & 12.37 & 27 & $16^{2}+8$ & 12.74 & 30 \\
$80^{2}+40$ & 12.39 & 28 & $20^{2}+10$ & 12.77 & 30 \\
\hline
\end{tabular}

\section{TABLE 7}

Discontinuous coefficient case for geometrically conforming subdomain partitions with $4 \times 4$ subregions. Left three columns: scalability as the increase of the subregion problem size $\widehat{N}$ when the subdomain problem sizes are fixed with $n=(H / h)=5$ or 6 . Right three columns: scalability as the increase of the subdomain problem size $n$ when the subregion problem sizes are fixed with $\widehat{N}=(\widehat{H} / H)=4$.

\begin{tabular}{|c||c|c||c||c|c|}
\hline \multicolumn{2}{|c||}{$4 \times 4$ subregions, $n$ fixed } & \multicolumn{3}{|c|}{$4 \times 4$ subregions, $\widehat{N}$ fixed } \\
\hline$\widehat{N}=\frac{\widehat{H}}{H}$ & Cond & Iter & $n=\frac{H}{h}$ & Cond & Iter \\
\hline 4 & 9.55 & 22 & $(5,4)$ & 9.55 & 22 \\
8 & 10.46 & 25 & $(10,8)$ & 13.01 & 26 \\
16 & 11.64 & 26 & $(20,16)$ & 16.90 & 30 \\
20 & 12.06 & 28 & $(25,20)$ & 18.24 & 31 \\
\hline
\end{tabular}

TABLE 8

Discontinuous coefficient case for geometrically nonconforming subdomain partitions with $4^{2}+2$ subregions. Left three columns: scalability as the increase of the subregion problem size $\widehat{N}$ when the subdomain problem sizes are fixed with $n=(H / h)=6,8$, or 10 . Right three columns: scalability as the increase of the subdomain problem size $n$ when the subregion problem sizes are fixed with $\widehat{N}=(\widehat{H} / H)=4$.

\begin{tabular}{|c||c|c||c||c|c|}
\hline \multicolumn{2}{|c||}{$4^{2}+2$ subregions, $n$ fixed } & \multicolumn{3}{c|}{$4^{2}+2$ subregions, $\widehat{N}$ fixed } \\
\hline$\widehat{N}=\frac{\widehat{H}}{H}$ & Cond & Iter & $n=\frac{H}{h}$ & Cond & Iter \\
\hline 4 & 11.87 & 27 & $(4,6,8)$ & 10.36 & 26 \\
8 & 12.23 & 28 & $(6,8,10)$ & 11.87 & 27 \\
16 & 12.74 & 30 & $(10,12,14)$ & 14.36 & 30 \\
20 & 13.04 & 31 & $(12,14,16)$ & 15.30 & 31 \\
\hline
\end{tabular}

size with the other mesh parameters fixed. We observe that the theoretical bound is still valid for the discontinuous coefficients in both the geometrically conforming and nonconforming subdomain partitions.

Acknowledgments. The authors are grateful to Professor Olof Widlund for all his help. They also thank the referees for useful comments and suggestions.

\section{REFERENCES}

[1] F. B. Belgacem And Y. Maday, The mortar element method for three dimensional finite elements, M2AN Math. Model. Numer. Anal., 31 (1997), pp. 289-302.

[2] F. B. Belgacem, The mortar finite element method with Lagrange multipliers, Numer. Math., 84 (1999), pp. 173-197. 
[3] C. Bernardi, Y. Maday, and A. T. Patera, A new nonconforming approach to domain decomposition: The mortar element method, in Nonlinear Partial Differential Equations and Their Applications. Collège de France Seminar, Vol. XI (Paris, 1989-1991), Pitman Res. Notes Math. 299, Longman Scientific and Technical, Harlow, 1994, pp. 13-51.

[4] S. C. Brenner And L.-Y. Sung, BDDC and FETI-DP without matrices or vectors, Comput. Methods Appl. Mech. Engrg., 196 (2007), pp. 1429-1435.

[5] S. C. Brenner, Korn's inequalities for piecewise $H^{1}$ vector fields, Math. Comp., 73 (2004), pp. 1067-1087.

[6] S. C. Brenner, A functional analytic framework for BDDC and FETI-DP, in Domain Decomposition Methods in Science and Engineering, Vol. XVII, Proceedings of the Seventeenth International Conference of Domain Decomposition Methods, Lect. Notes Comput. Sci. Eng. 60, U. Langer, M. Discacciati, D. Keyes, O. Widlund, and W. Zulehner, eds., Springer-Verlag, Berlin, 2008, pp. 239-246.

[7] Y.-W. Chang, H. H. Kim, And C.-O. Lee, Preconditioners for the dual-primal FETI methods on nonmatching grids: Numerical study, Comput. Math. Appl., 51 (2006), pp. 697-712.

[8] L. C. Cowsar, J. Mandel, And M. F. Wheeler, Balancing domain decomposition for mixed finite elements, Math. Comp., 64 (1995), pp. 989-1015.

[9] C. R. Dohrmann, A preconditioner for substructuring based on constrained energy minimization, SIAM J. Sci. Comput., 25 (2003), pp. 246-258.

[10] C. R. Dohrmann, A Substructuring Preconditioner for Nearly Incompressible Elasticity Problems, Technical report SAND2004-5393, Sandia National Laboratories, Albuquerque, NM, 2004.

[11] C. R. Dohrmann, An approximate BDDC preconditioner, Numer. Linear Algebra Appl., 14 (2007), pp. 149-168.

[12] C. Kim, R. D. Lazarov, J. E. Pasciak, and P. S. Vassilevski, Multiplier spaces for the mortar finite element method in three dimensions, SIAM J. Numer. Anal., 39 (2001), pp. 519-538.

[13] H. H. Kim, M. Dryja, And O. B. Widlund, A BDDC method for mortar discretizations using a transformation of basis, SIAM J. Numer. Anal., 47 (2008), pp. 136-157.

[14] H. H. KIM, A BDDC algorithm for mortar discretization of elasticity problems, SIAM J. Numer. Anal., 46 (2008), pp. 2090-2111.

[15] A. Klawonn, O. Rheinbach, And O. B. Widlund, An analysis of a FETI-DP algorithm on irregular subdomains in the plane, SIAM J. Numer. Anal., 46 (2008), pp. 2484-2504.

[16] A. Klawonn and O. Rheinbach, Inexact FETI-DP methods, Internat. J. Numer. Methods Engrg., 69 (2007), pp. 284-307.

[17] A. Klawonn and O. B. Widlund, Dual-primal FETI methods for linear elasticity, Comm. Pure Appl. Math., 59 (2006), pp. 1523-1572.

[18] J. Li And X. Tu, Convergence Analysis of a Balancing Domain Decomposition Method for Solving Interior Helmholtz Equations, Numer. Linear Algebra Appl., to appear.

[19] J. Li AND O. WidLund, BDDC algorithms for incompressible Stokes equations, SIAM J. Numer. Anal., 44 (2006), pp. 2432-2455.

[20] J. Li And O. Widlund, FETI-DP, BDDC, and block Cholesky methods, Internat. J. Numer. Methods Engrg., 66 (2006), pp. 250-271.

[21] J. Li AND O. WIDLUND, On the use of inexact subdomain solvers for BDDC algorithms, Comput. Methods Appl. Mech. Engrg., 196 (2007), pp. 1415-1428.

[22] J. Mandel, C. R. Dohrmann, and R. Tezaur, An algebraic theory for primal and dual substructuring methods by constraints, Appl. Numer. Math., 54 (2005), pp. 167-193.

[23] J. Mandel And C. R. Dohrmann, Convergence of a balancing domain decomposition by constraints and energy minimization, Numer. Linear Algebra Appl., 10 (2003), pp. 639-659.

[24] M. SARKIS, Nonstandard coarse spaces and Schwarz methods for elliptic problems with discontinuous coefficients using non-conforming elements, Numer. Math., 77 (1997), pp. 383-406.

[25] D. Stefanica, Domain Decomposition Methods for Mortar Finite Elements, Ph.D. thesis, Department of Computer Science, Courant Institute, New York Unversity, New York, 2000.

[26] A. Toselli And O. Widlund, Domain Decomposition Methods-Algorithms and Theory, Springer Ser. Comput. Math. 34, Springer-Verlag, Berlin, 2005.

[27] X. TU And J. LI, A balancing domain decomposition method by constraints for advectiondiffusion problems, Commun. Appl. Math. Comput. Sci., 3 (2008), pp. 25-60.

[28] X. TU, A BDDC algorithm for a mixed formulation of flows in porous media, Electron. Trans. Numer. Anal., 20 (2005), pp. 164-179.

[29] X. Tu, BDDC Domain Decomposition Algorithms: Methods with Three Levels and for Flow in Porous Media, Ph.D. thesis, Courant Institute, New York University, New York, 2006.

[30] X. TU, A BDDC algorithm for flow in porous media with a hybrid finite element discretization, Electron. Trans. Numer. Anal., 26 (2007), pp. 146-160.

Copyright $@$ by SIAM. Unauthorized reproduction of this article is prohibited. 
[31] X. Tu, Three-level BDDC in three dimensions, SIAM J. Sci. Comput., 29 (2007), pp. 17591780.

[32] X. Tu, Three-level BDDC in two dimensions, Internat. J. Numer. Methods Engrg., 69 (2007), pp. 33-59.

[33] B. I. Wohlmuth, A mortar finite element method using dual spaces for the Lagrange multiplier, SIAM J. Numer. Anal., 38 (2000), pp. 989-1012.

[34] B. I. Wohlmuth, Discretization Methods and Iterative Solvers Based on Domain Decomposition, Lect. Notes Comput. Sci. Eng. 17, A. Toselli and O. Widlund, eds., Springer-Verlag, Berlin, 2001.

Copyright (c) by SIAM. Unauthorized reproduction of this article is prohibited. 